\title{
Alveolar macrophages from persons living with HIV show impaired epigenetic response to Mycobacterium tuberculosis
}

\author{
Wilian Correa-Macedo, ${ }^{1,2,3}$ Vinicius M. Fava, ${ }^{2,3}$ Marianna Orlova, ${ }^{2,3}$ Pauline Cassart, ${ }^{2,3}$ Ron Olivenstein, ${ }^{4}$ Joaquin Sanz, ${ }^{5}$ \\ Yong Zhong Xu, ${ }^{2,3}$ Anne Dumaine, ${ }^{6}$ Renata H.M. Sindeaux, ${ }^{7}$ Vania Yotova, ${ }^{7}$ Alain Pacis, ${ }^{8}$ Josée Girouard, ${ }^{2,9}$ Barbara Kalsdorf, ${ }^{10,11}$ \\ Christoph Lange, ${ }^{10,11,12,13,14}$ Jean-Pierre Routy, ${ }^{2,9}$ Luis B. Barreiro, ${ }^{6}$ and Erwin Schurr ${ }^{1,2,3}$ \\ 'Department of Biochemistry, Faculty of Medicine, McGill University, Montreal, Quebec, Canada. ${ }^{2}$ Program in Infectious Diseases and Global Health, The Research Institute of the McGill University Health \\ Centre, Montréal, Quebec, Canada. ${ }^{3}$ McGill International TB Centre, Department of Medicine, Faculty of Medicine, McGill University, Montreal, Quebec, Canada. ${ }^{4}$ Translational Research in Respiratory \\ Diseases Program, The Research Institute of the McGill University Health Centre, Montreal, Quebec, Canada. Institute for Bio-computation and Physics of Complex Systems BIFI, Department of Theoretical

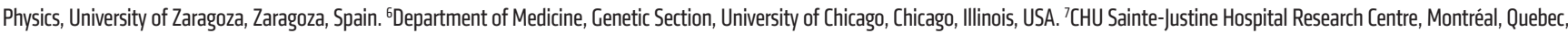 \\ Canada. ${ }^{8}$ Canadian Centre for Computational Genomics, McGill University and Genome Quebec Innovation Center, Montréal, Quebec, Canada. ${ }^{9}$ Chronic Viral Illnesses Service and Division of Hematology, \\ McGill University Health Centre, Montréal, Quebec, Canada. ${ }^{10}$ Research Center Borstel, Clinical Infectious Diseases, Borstel, Cermany. ${ }^{11}$ Cluster of Excellence Precision Medicine in Chronic Inflammation, Kiel, \\ Germany. ${ }^{12}$ Cerman Center for Infection Research (DZIF) Tuberculosis Unit, Borstel, Cermany. ${ }^{13}$ Respiratory Medicine \& International Health, University of Lübeck, Lübeck, Cermany. ${ }^{14}$ Clobal TB Program, \\ Baylor College of Medicine and Texas Children's Hospital, Houston, Texas, USA.
}

Persons living with HIV (PLWH) are at increased risk of tuberculosis (TB). HIV-associated TB is often the result of recent infection with Mycobacterium tuberculosis (M. tuberculosis) followed by rapid progression to disease. Alveolar macrophages (AMs) are the first cells of the innate immune system that engage M. tuberculosis, but how HIV and antiretroviral therapy (ART) affect the anti-mycobacterial response of AMs is not known. To investigate the impact of HIV and ART on the transcriptomic and epigenetic response of AMs to $M$. tuberculosis, we obtained AMs by bronchoalveolar lavage from 20 PLWH receiving ART, 16 control subjects who were HIV-free (HC), and 14 subjects who received ART as preexposure prophylaxis (PrEP) to prevent HIV infection. Following in vitro challenge with M. tuberculosis, AMs from each group displayed overlapping but distinct profiles of significantly up- and downregulated genes in response to $M$. tuberculosis. Comparatively, AMs isolated from both PLWH and PrEP subjects presented a substantially weaker transcriptional response. In addition, AMs from HC subjects challenged with $M$. tuberculosis responded with pronounced chromatin accessibility changes while AMs obtained from PLWH and PrEP subjects displayed no significant changes in their chromatin state. Collectively, these results revealed a stronger adverse effect of ART than HIV on the epigenetic landscape and transcriptional responsiveness of AMs.

\section{Introduction}

Globally, tuberculosis (TB) is the leading cause of death due to a single bacterial pathogen. In 2019, there were an estimated 1.4 million deaths caused by TB, which included 208,000 people living with the human immunodeficiency virus (HIV) (1). Of the estimated 10 million people who fell ill with TB, 820,000 were persons living with HIV (PLWH), consistent with a 2.0-fold higher mortality due to TB in HIV-positive relative to HIV-negative persons. Globally, the proportion of notified HIV-positive TB cases on antiretroviral therapy was $88 \%$ with large gaps of antiretrovi-

Related Commentary: https://doi.org/10.1172/JCI154407

Authorship note: WCM and VMF are co-first authors. LBB and ES are co-senior authors.

Conflict of interest: The authors have declared that no conflict of interest exists. Copyright: @ 2021, American Society for Clinical Investigation.

Submitted: January 25, 2021; Accepted: August 26, 2021; Published: November 15, 2021

Reference information: J Clin Invest. 2021;131(22):e148013.

https://doi.org/10.1172/JCl148013. ral therapy (ART) coverage among countries (1). However, even people on long-term ART still have substantially increased risk of developing TB (2), which is usually the result of new infection rather than reactivation of latent $\mathrm{TB}(3-6)$.

Transmission of Mycobacterium tuberculosis (M. tuberculosis) occurs by aerosols that are inhaled by an exposed person. Results from the mouse model show that after reaching the lung alveoli, M. tuberculosis bacilli are being rapidly taken up by alveolar macrophages (AMs). Following a delay of approximately 10 days, successful establishment of infection occurs once $M$. tuberculosisinfected AMs traverse the airway epithelium and establish themselves in the lung interstitium. There, transfer of $M$. tuberculosis to permissive inflammatory macrophages occurs and $\mathrm{T}$ cell priming is initiated in the draining lymph nodes (7). However, much of the AM-M. tuberculosis interaction in the alveoli remains unknown.

In humans, not each exposure results in successful infection, and a subset of highly exposed persons entirely resist infection, as inferred from the absence of $\mathrm{CD}^{+} \mathrm{T}$ cell anti-M. tuberculosis immunity $(8,9)$. Hence it seems possible that human AMs are capable of limiting transfer of inhaled $M$. tuberculosis bacilli to the 
lung interstitium. The increased risk of $M$. tuberculosis infection and TB disease for PLWH may therefore reflect an impaired ability of AMs to restrict M. tuberculosis to the alveoli, thus placing early events in the $M$. tuberculosis-host interaction as a central aspect of $\mathrm{TB}$ resistance.

Based on the above considerations, we decided to investigate the transcriptome and epigenome of the ex vivo AM-M. tuberculosis interaction employing AMs obtained from PLWH and HIVnegative participants. Since ART is given to most PLWH, we also included an additional control group consisting of persons who are HIV-negative receiving ART for preexposure prophylaxis (PrEP).

\section{Results}

Study subjects. To investigate the influence of HIV on the ability of AMs to mount a response to $M$. tuberculosis infection, we studied 3 groups of subjects: PLWH receiving ART $(n=20)$, persons without HIV but receiving ART as $\operatorname{PrEP}(n=14)$, and persons without HIV not receiving $\operatorname{PrEP}(\mathrm{HC} ; n=16$; Table 1 and Supplemental Table 1; supplemental material available online with this article; https:/doi. org/10.1172/JCI148013DS1). All PrEP subjects were on continuous PrEP. While we did not enroll newly diagnosed patients with HIV before the onset of ART, we note that globally a plurality of PLWH are on ART (10). Consequently, PLWH receiving ART are more representative of the impact of HIV on TB than PLWH who are not receiving ART. We isolated AMs by BAL from all subjects and challenged the cells in vitro with M. tuberculosis for 18 to 20 hours. RNA and DNA were isolated from uninfected and M. tuberculosischallenged cells and used to assess gene expression levels by RNASeq, chromatin structure by ATAC-Seq, and presence of the histone activation mark H3K27ac by ChIP-Seq (Figure 1A). However, due to different quality control procedures and variable numbers of AMs obtained per subject, different counts of subjects were used for the transcriptomics and epigenetics experiments (Figure 1A).

$A M s$ from PLWH and PrEP subjects display blunted $m R N A$ transcriptomic changes in response to $M$. tuberculosis. Across the 3 phenotypic groups, we identified 1626 differentially expressed genes (DEGs) in response to M. tuberculosis (Supplemental Table 2). However, there were pronounced DEG count differences in their transcriptional responses (Figure 1B). AMs from HC subjects showed 1434 DEGs in response to M. tuberculosis as compared with only 679 and 255 DEGs in PLWH and PrEP subjects, respectively (Figure 1B and Supplemental Figure 1A). The magnitude of AM transcriptional response to $M$. tuberculosis also differed among the 3 groups. The smallest mean absolute $\log _{2}$ fold change $\left(\log _{2} \mathrm{FC}\right)$ in response to $M$. tuberculosis infection was observed among PrEP subjects while HC subjects displayed the strongest transcriptional response (Figure 1C and Supplemental Figure 1A). PLWH displayed a stronger transcriptional response than PrEP subjects, suggesting an interaction of HIV and ART in PLWH (Figure 1C and Supplemental Figure 1B). To test if M. tuberculosis effects in PLWH and PrEP subjects were weaker for all DEGs or only for specific gene sets, we assessed the correlations of $M$. tuberculosis-triggered $\log _{2} \mathrm{FC}$ across the 3 groups. We observed that $\log _{2} \mathrm{FC}$ values were strongly correlated among all 3 groups, but were consistently higher for corresponding genes from HC subjects (Supplemental Figure 1B), which suggested a general transcriptional impairment in cells from PLWH and PrEP subjects.
Pathway and GO-term enrichment analysis revealed that $M$. tuberculosis-induced transcriptional changes were enriched in biological functions representative of macrophage anti-mycobacterial host responses, such as interferon (IFN) and Toll-like receptor signaling pathways (Figure 1D). However, compared with HC subjects, the number of significant GO terms/pathways was substantially lower for the PLWH and PrEP groups (Supplemental Table 3). Moreover, even among shared significant terms, the cumulative transcriptional response of genes in these terms was stronger in the HC group, which further emphasized the blunted antimycobacterial response by PLWH and PrEP subjects (Figure 1D). We also tested which genes had a significantly different magnitude of response to M. tuberculosis among phenotype groups (Figure 2A and Supplemental Table 2). We identified 333 genes significantly different between the groups (Supplemental Table 4) with the majority displaying lower upregulation in response to $M$. tuberculosis for PLWH and PrEP groups relative to the HC group (Supplemental Figure 1, C and D and Supplemental Table 3). These results provided a formal confirmation that the transcriptional response to $M$. tuberculosis challenge by AMs of $\mathrm{HC}$ was more vigorous compared with the one mounted by PrEP subjects and PLWH. At baseline, mean gene expression levels were very similar between HC subjects, PrEP subjects, and PLWH (Supplemental Figure 2). This observation suggested that differences in the magnitude of the response to $M$. tuberculosis were not driven by a higher baseline gene expression in any of the tested groups.

Of note, 40 genes were differentially expressed at FDR less than or equal to $5 \%$ in response to $M$. tuberculosis between the PLWH and PrEP groups (Figure 2A and Supplemental Table 5). Among these 40 genes were CD209 (alias DC_SIGN) and OTUD3, 2 genes intimately involved in the anti-HIV and anti-M. tuberculosis host response (11-16). M. tuberculosis infection triggered upregulation of CD2O9 in the HC and PrEP groups while AMs from PLWH did not display significant change in expression levels. Conversely, in response to $M$. tuberculosis, the antiviral mediator OTUD3 was upregulated in PLWH and downregulated in the PrEP group. These observations suggested that HIV effected potentially biologically relevant changes on AM physiology that were not overcome by the broader ART-linked dampening of transcriptional responsiveness.

To explore if the group differences were mainly driven by strong outliers, we derived a per subject average $\log _{2} \mathrm{FC}$ across DEGs in significant pathways/GO terms. We found that AMs from HC subjects displayed a more pronounced group transcriptional response to $M$. tuberculosis. For example, the mean $\log _{2} \mathrm{FC}$ for the term "I-kappaB kinase/NF-kappaB signaling" was 3.22-fold ( $P=$ $\left.2.4 \times 10^{-3}\right)$ and 2.39-fold $\left(P=3.8 \times 10^{-2}\right)$ higher in HC subjects as compared with PLWH and PrEP subjects, respectively (Figure 2B). GO terms and pathways tagged by the DEGs from PLWH and PrEP versus $\mathrm{HC}$ subjects, which were significantly more induced in AMs from $\mathrm{HC}$ subjects, revealed a predominance of innate-immune and intracellular defense response terms (Figure 2C). This observation alongside the exclusion of possible outlier-driven group differences supported the reduced ability of AMs from PLWH and PrEP subjects to mount an effective anti-mycobacterial transcriptional response to challenge with $M$. tuberculosis. Finally, we also measured M. tuberculosis-triggered secretion of 7 cytokines and 
Table 1. Demographics and characteristics of enrolled subjects

\begin{tabular}{|c|c|c|c|c|c|c|c|c|c|c|c|c|c|}
\hline $\begin{array}{l}\text { Sample } \\
\text { ID }\end{array}$ & Sex & Ethnicity ${ }^{A}$ & $\begin{array}{c}\text { Age at } \\
\text { recruitment, } \\
\text { years }\end{array}$ & Smoker & $\begin{array}{l}\text { Recreational } \\
\text { drug }\end{array}$ & $\begin{array}{l}\text { Medical } \\
\text { history }\end{array}$ & ART & Prescription drug molecules & $\begin{array}{c}\text { Sexually } \\
\text { transmitted } \\
\text { infections }\end{array}$ & $\begin{array}{c}\text { CD4 } \\
\text { count }\end{array}$ & $\begin{array}{l}\text { RNA- } \\
\text { Seq }\end{array}$ & $\begin{array}{c}\text { ATAC- } \\
\text { Seq }\end{array}$ & $\begin{array}{l}\text { ChIP- } \\
\text { Seq }\end{array}$ \\
\hline AMC2 & M & White & 59 & Yes & Cannabis & None & No & None & None & 495 & Yes & Yes & No \\
\hline AMC7 & M & White & 49 & No & No & HTN, T1DM, gout & No & None & None & 500 & Yes & Yes & No \\
\hline AMC8 & M & White & 25 & No & No & None & No & None & None & 1536 & Yes & No & No \\
\hline AMC9 & M & White & 58 & Yes & Cannabis & None & No & None & None & 1290 & Yes & Yes & Yes \\
\hline AMC10 & M & White & 29 & No & No & None & No & None & None & 1091 & Yes & Yes & Yes \\
\hline AMC11 & M & White & 27 & No & No & None & No & None & None & 439 & Yes & Yes & Yes \\
\hline AMC12 & M & White & 40 & No & No & None & No & None & None & 1263 & Yes & Yes & Yes \\
\hline AMC13 & $\mathrm{F}$ & White & 31 & No & No & None & No & None & None & 871 & Yes & Yes & Yes \\
\hline AMC14 & $\mathrm{F}$ & White & 40 & Yes & No & None & No & None & None & 1023 & Yes & Yes & Yes \\
\hline AMC15 & $\mathrm{F}$ & White & 41 & No & No & None & No & None & None & 843 & Yes & Yes & No \\
\hline AMC16 & $\mathrm{F}$ & White & 37 & No & No & None & No & None & None & 1249 & Yes & Yes & No \\
\hline AMC18 & M & White & 27 & No & No & None & No & None & None & 826 & Yes & Yes & No \\
\hline AMC19 & $M$ & White & 53 & No & No & None & No & None & None & 692 & Yes & Yes & No \\
\hline AMC20 & M & Asian & 34 & No & No & None & No & None & None & 545 & Yes & No & No \\
\hline AMC21 & M & White & 57 & No & No & HTN, T2DM & No & None & None & 1077 & Yes & Yes & No \\
\hline AMC22 & $\mathrm{F}$ & White & 42 & Yes & Cannabis & None & No & None & None & 1248 & Yes & Yes & Yes \\
\hline AMP23 & M & White & 65 & Yes & Cannabis & Fibromyalgia & PrEP (2017) & Emtricitabine, Tenofovir_DF & None & 819 & Yes & No & No \\
\hline AMP25 & M & White & 28 & No & No & ADHD, depression & $\operatorname{PrEP}(2016)$ & Emtricitabine, Tenofovir_DF & None & 575 & Yes & No & No \\
\hline AMP26 & M & White & 25 & No & Cannabis & Chronic alcoholism & $\operatorname{PrEP}(2015)$ & Emtricitabine, Tenofovir_DF & Syphilis & 835 & Yes & No & No \\
\hline AMP27 & M & Hispanic & 36 & No & No & None & $\operatorname{PrEP}(2014)$ & Emtricitabine, Tenofovir_DF & $\begin{array}{l}\text { Chlamydia and } \\
\text { gonorrhea }\end{array}$ & 961 & Yes & Yes & No \\
\hline AMP28 & M & White & 60 & Yes & No & HTN, T1DM, ADHD & $\operatorname{PrEP}(2016)$ & Emtricitabine, Tenofovir_DF & None & 1254 & Yes & Yes & Yes \\
\hline AMP29 & M & North African & 37 & Yes & Cannabis & None & $\operatorname{PrEP}(2013)$ & Emtricitabine, Tenofovir_DF & Chlamydia & 981 & Yes & Yes & No \\
\hline AMP30 & M & White & 30 & No & No & Depression & $\operatorname{PrEP}(2016)$ & Emtricitabine, Tenofovir_DF & None & 847 & Yes & No & No \\
\hline AMP31 & M & White & 36 & No & No & None & $\operatorname{PrEP}(2016)$ & Emtricitabine, Tenofovir_DF & None & 1163 & Yes & Yes & No \\
\hline AMP32 & M & White & 36 & No & No & None & $\operatorname{PrEP}(2017)$ & Emtricitabine, Tenofovir_DF & None & 1069 & Yes & Yes & No \\
\hline AMP33 & M & White & 28 & No & Speed & Depression & $\operatorname{PrEP}(2019)$ & Emtricitabine, Tenofovir_DF & None & 691 & Yes & Yes & Yes \\
\hline AMP34 & M & Asian & 42 & No & No & None & $\operatorname{PrEP}(2016)$ & Emtricitabine, Tenofovir_DF & Chlamydia & 208 & Yes & Yes & Yes \\
\hline AMP35 & M & White & 25 & Yes & Cocaine & None & $\operatorname{PrEP}(2018)$ & Emtricitabine, Tenofovir_DF & Chlamydia & 832 & Yes & Yes & Yes \\
\hline AMP36 & M & White & 28 & Yes & Ketamine & None & $\operatorname{PrEP}(2016)$ & Emtricitabine, Tenofovir_DF & Gonorrhea & 1009 & Yes & Yes & Yes \\
\hline AMP37 & M & White & 40 & No & Cannabis & None & $\operatorname{PrEP}(2017)$ & Emtricitabine, Tenofovir_DF & None & 1454 & Yes & Yes & Yes \\
\hline AMV3 & M & White & 52 & No & No & HIV (1996) & ART (1998) & Emtricitabine, Tenofovir_DF, Raltegravir & None & 382 & Yes & Yes & No \\
\hline AMV4 & M & White & 56 & Yes & Cannabis & HIV (2002) & ART (2002) & Emtricitabine, Tenofovir_AF, Bictegravir & None & 1172 & Yes & Yes & Yes \\
\hline AMV5 & M & White & 59 & No & No & HIV (1995) & ART (2009) & Emtricitabine, Tenofovir_DF, Raltegravir & None & 606 & Yes & Yes & No \\
\hline AMV8 & M & White & 46 & No & No & HIV (2002) & ART (2013) & Emtricitabine, Tenofovir_DF, Rilpivirine & None & 533 & Yes & No & No \\
\hline AMV9 & M & White & 56 & Yes & Cannabis & HIV (2002) & ART (2004) & Emtricitabine, Tenofovir_DF, Raltegravir & None & 536 & Yes & Yes & Yes \\
\hline AMV10 & M & White & 60 & No & No & HIV (1992) & ART (1996) & $\begin{array}{c}\text { Etravirine, Darunavir, Dolutegravir, } \\
\text { Ritonavir }\end{array}$ & None & 269 & Yes & Yes & No \\
\hline AMV11 & M & White & 50 & Yes & No & HIV (1997) & ART (1999) & Emtricitabine, Tenofovir_DF, Rilpivirine & None & 439 & Yes & Yes & Yes \\
\hline AMV13 & $\mathrm{F}$ & White & 54 & Yes & Cannabis & HIV (1994) & ART (1994) & $\begin{array}{c}\text { Emtricitabine, Tenofovir_AF, } \\
\text { Dolutegravir, Rilpivirine }\end{array}$ & None & 716 & Yes & Yes & Yes \\
\hline AMV14 & M & White & 57 & Yes & Cannabis & HIV (1996) & ART (1997) & $\begin{array}{l}\text { Abacavir, Lamivudine, } \\
\text { Atazanavir, Ritonavir }\end{array}$ & None & 983 & Yes & Yes & Yes \\
\hline AMV15 & M & White & 51 & No & No & HIV (2003) & ART (2004) & Abacavir, Dolutegravir, Lamivudine & None & 468 & Yes & Yes & Yes \\
\hline AMV16 & M & White & 56 & Yes & No & HIV (1987) & ART (2002) & $\begin{array}{l}\text { Emtricitabine, Tenofovir_AF, } \\
\text { Cobicistat, Elvitegravir }\end{array}$ & None & 1005 & Yes & No & Yes \\
\hline AMV17 & M & White & 41 & Yes & Cannabis & HIV (2014) & ART (2015) & Tenofovir_DF, Dolutegravir, Rilpivirine & None & 1267 & Yes & Yes & Yes \\
\hline AMV18 & M & White & 40 & Yes & Cannabis & HIV (2016) & ART (2016) & Tenofovir_DF, Dolutegravir, Lamivudine & None & 989 & Yes & No & No \\
\hline AMV19 & M & White & 57 & No & Cannabis & HIV (2005) & ART (2006) & Abacavir, Dolutegravir, Lamivudine & None & 565 & Yes & Yes & No \\
\hline AMV20 & M & White & 58 & No & Cannabis & HIV (1997) & ART (1997) & $\begin{array}{l}\text { Emtricitabine, Tenofovir_DF, } \\
\text { Cobiscistat, Darunavir }\end{array}$ & None & 727 & Yes & Yes & No \\
\hline AMV21 & M & White & 62 & Yes & Cannabis & HIV (2012) & ART (2012) & Emtricitabine, Tenofovir_DF, Dolutegravir & None & 650 & Yes & Yes & Yes \\
\hline AMV23 & M & White & 33 & Yes & Cannabis & HIV (2017) & ART (2017) & $\begin{array}{l}\text { Emtricitabine, Tenofovir_AF, } \\
\text { Cobicistat, Elvitegravir }\end{array}$ & None & 633 & Yes & Yes & Yes \\
\hline AMV25 & M & White & 60 & No & No & HIV (2006) & ART (2007) & Emtricitabine, Tenofovir_DF, Efavirenz & None & 906 & Yes & Yes & Yes \\
\hline AMV26 & M & White & 50 & No & No & HIV (2000) & ART (2010) & Emtricitabine, Tenofovir_DF, Efavirenz & None & 471 & Yes & Yes & No \\
\hline AMV27 & M & White & 59 & No & No & HIV (2017) & ART (2017) & Emtricitabine, Tenofovir_AF, Raltegravir & None & 501 & Yes & Yes & No \\
\hline
\end{tabular}

${ }^{A}$ Ethnicity was self-declared by participants. HTN, hypertension; T1DM, type 1 diabetes mellitus; T2DM, type 2 diabetes mellitus; ADHD, attention deficit/hyperactivity disorder. 
A

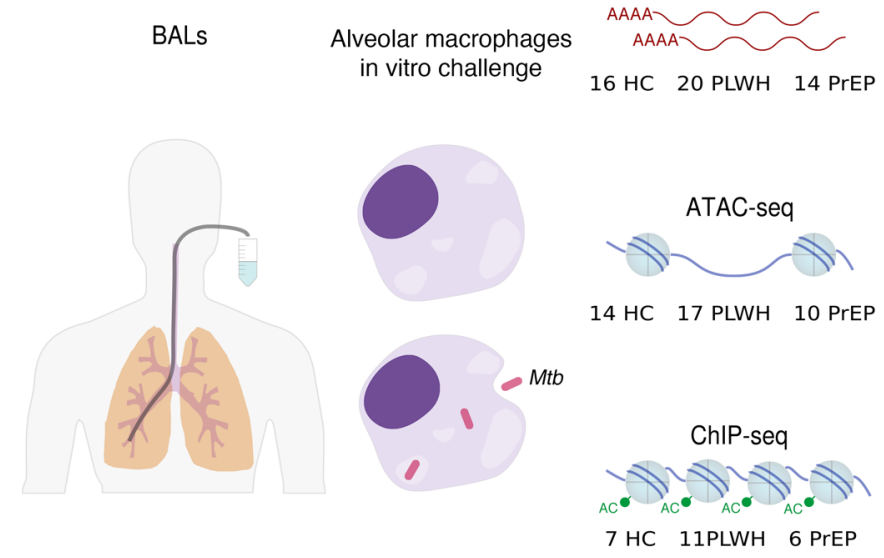

B
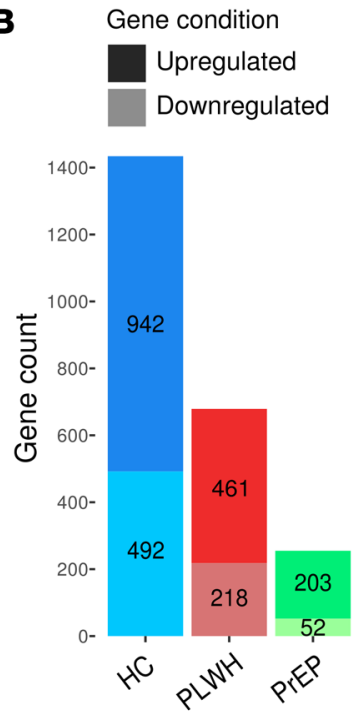

C

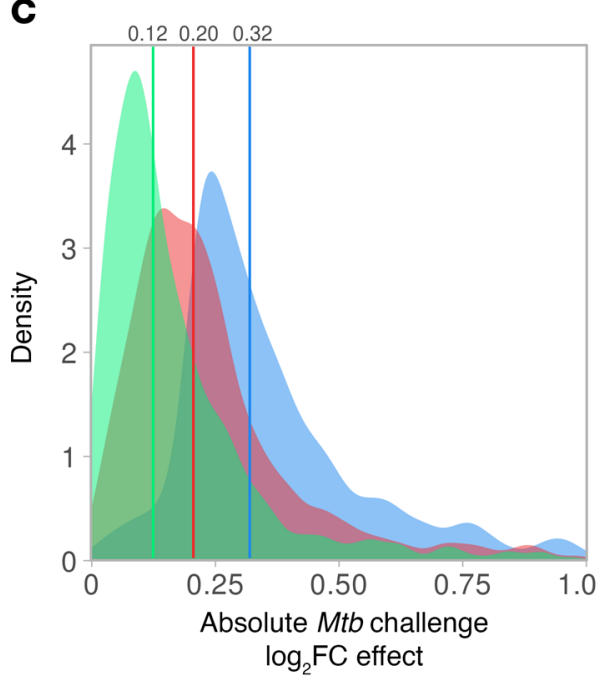

D

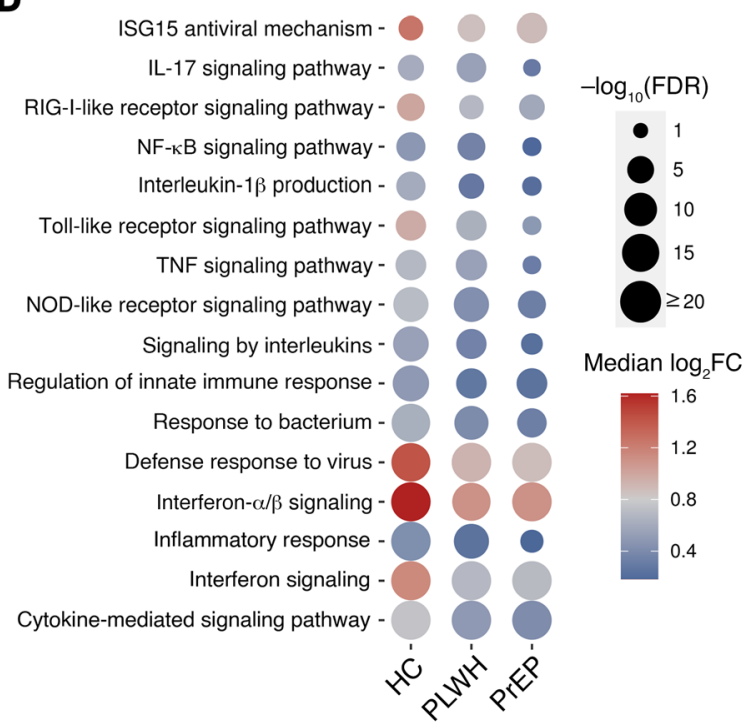

Figure 1. Study design and differential gene expression by AMs in response to M. tuberculosis. (A) Experimental design and sample number by group. Each subject underwent BAL. AMs were obtained from BAL and challenged in vitro with M. tuberculosis for 18 to 20 hours. RNA was obtained for RNA-Seq, whereas DNA was used for ATAC-Seq and ChIP-Seq experiments. (B) Bar graph summarizing DEGs by AM, following M. tuberculosis challenge across the 3 phenotypic groups (HCs; PLWH on antiretroviral therapy; PrEP subjects). The $y$ axis indicates the cumulative DEC count and group ID is indicated below each bar. Dark color shades represent upregulated genes (positive log fold-change; $\log _{2} \mathrm{FC}$ ) whereas light shades depict downregulated genes (negative $\log _{2} F C$ ). (C) Density plot presenting $\log _{2} F C$ as absolute values. All DEGs from $B$ had their $\log _{2} F C$ converted to absolute values and plotted using density function. Vertical colored lines indicate the median of absolute values per group, with exact values given on top. Blue shade represents $\mathrm{HC}$ subjects, red indicates PLWH, and green indicates PrEP subjects. (D) Dot plot presenting significance and overall effect for selected pathways and GO terms. Pathways/GO terms are listed on the left, dot sizes reflect the negative $\log _{10}$ FDR values for the enrichment test (bigger dots have smaller $P$ values) and colors from red (higher value) to blue (lower value) indicate the median $\log _{2} \mathrm{FC}$ across all detected genes in the corresponding pathway or $\mathrm{GO}$ term. To derive the average $\log _{2} F C$ for a pathway/GO term, for each group we identified the corresponding DEGs, pooled all gene IDs from the 3 groups, retrieved the log 2 FC for each of these genes, and established the median of $\log _{2} \mathrm{FC}$ per group.

found that AMs from the PLWH and PrEP groups show reduced cytokine secretion levels compared with AMs from the HC group (Supplemental Figure 3).

M. tuberculosis does not induce chromatin remodeling in AMs from PLWH and PrEP subjects. Next, we evaluated M. tuberculosis-triggered epigenetic changes of AM chromatin accessibility and H3K27 acetylation. Analysis of differentially open chromatin (DOC) in response to M. tuberculosis indicated that AMs from HC subjects had significant increased accessibility (opening) in 8389 regions and repression (closing) in 3971 regions (Figure 3A and Supplemental Tables 6 and 7). In contrast, AMs isolated from PLWH or PrEP subjects displayed near-complete absence of significant chromatin remodeling in response to M. tuberculosis (Figure 3, A and B). Pathway and GO-term enrichment analyses of genes annotated to DOC in HC identified typical viral and bacterial response terms (Figure 3, C and D). A broad-based epigenetic impairment was confirmed for AMs from PLWH and PrEP subjects by tracking regions with differently H3K27 acetyl- 
A
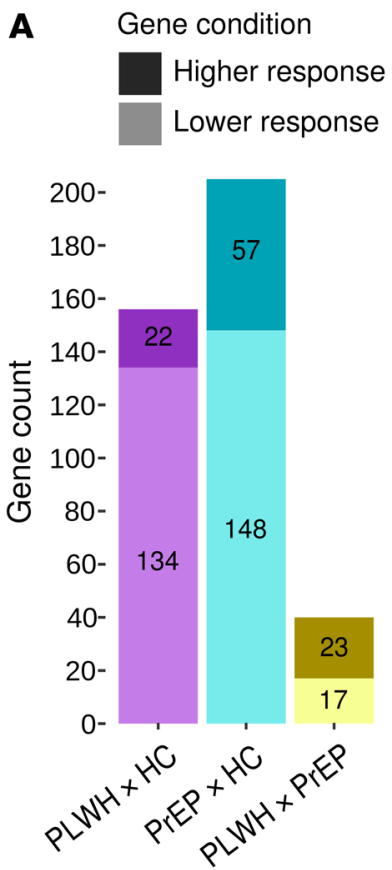

B

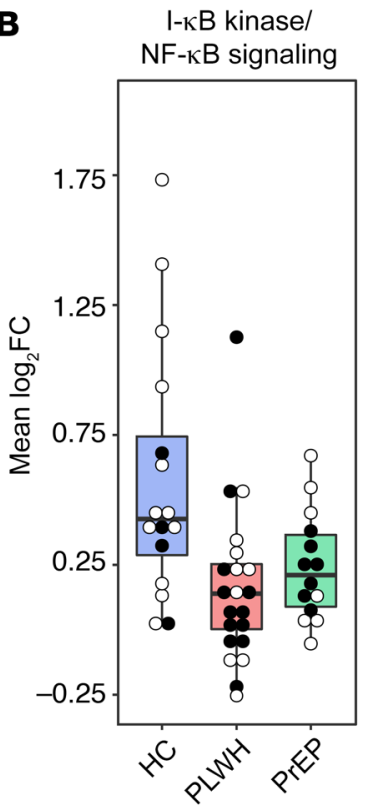

NOD-like receptor signaling pathway

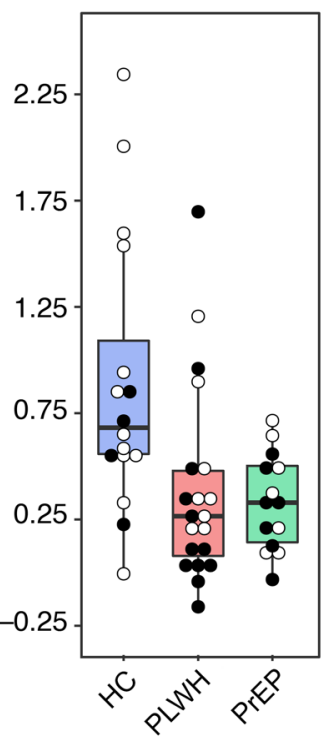

Interferon signaling

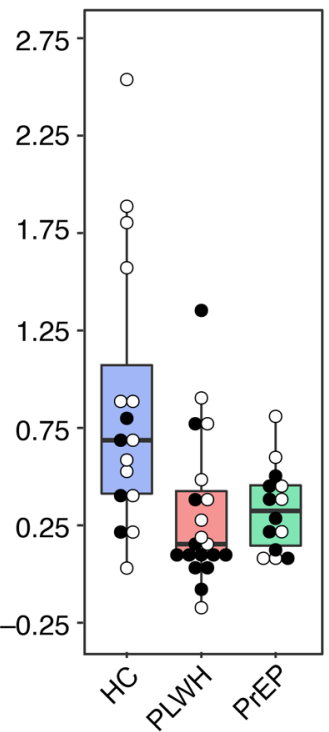

C

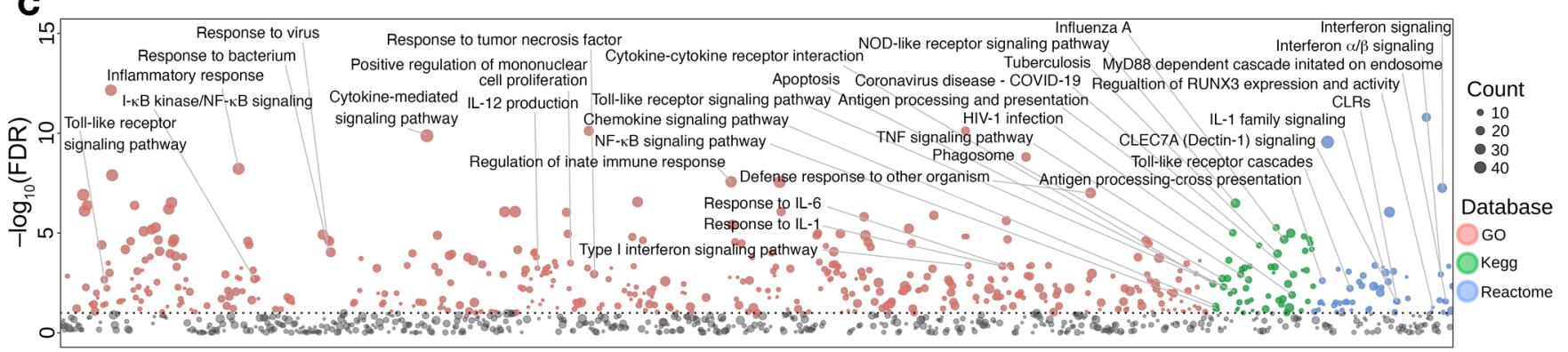

Figure 2. AMs display significant differences between groups in transcriptomic response to M. tuberculosis. (A) Bar graph summarizing genes that displayed significantly different $\log _{2} \mathrm{FC}$ between groups (differential M. tuberculosis response). The $y$ axis indicates the cumulative DEC count whereas the $x$ axis indicates the pair-wise group contrast. Dark color shading represents genes with higher response (e.g., M. tuberculosis effect in PLWH vs HC subjects resulted in a positive $\log _{2}$ FC difference) while light shading depicts genes with lower response (negative $\log _{2}$ FC difference). The union of all gene IDs identified across the 3 contrasts $(n=401)$ resulted in 333 unique gene IDs. (B) Boxplot for 3 selected pathway/GO terms for genes with significant differential M. tuberculosis response between PLWH or PrEP subjects against controls $(n=302)$. For each term, the $y$ axis displays the mean log $F C$ and dots represent per-subject mean value for pooled DEGs. Phenotypic groups are indicated below each box plot across the 3 terms. White dots represent mean log $2 \mathrm{FC}$ for subjects who do not smoke cigarettes or who have used cannabis, whereas black dots depict smokers and/or cannabis users. To derive the subject mean $\log _{2} \mathrm{FC}$, we used pooled DEGs from the first 2 columns in $\mathbf{A}$ which were significant for interaction contrasts between PLWH vs HC subjects and PrEP subjects versus $\mathrm{HC}$ subjects, obtained subject-wise $\log _{2} \mathrm{FC}$ for each DEC and averaged the $\log _{2} \mathrm{FC}$ from all genes per subject. Results showed that group differences are not driven by outliers and that smoking or cannabis consumption do not explain group differences. (C) Manhattan plot for enrichment test of pathways and GO terms from merged DEGs detected in the differential M. tuberculosis responses of PLWH vs HC subjects and PrEP subjects versus HC subjects. The $y$ axis indicates the negative $\log _{10}$ FDR values whereas the tested terms are arranged along the $x$ axis. The horizontal dashed line represents the 10\% FDR cut-off for significant pathways/CO terms. Dots are sized as a function of the DEG number in a term and colors represent the database from which the terms were obtained. Identified terms represent innate immune processes and intracellular defense mechanisms. C-type lectin receptors, CLRs.

ation (DAc), a mark of active chromatin (Figure $4, \mathrm{~A}$ and $\mathrm{B}$ and Supplemental Tables 6 and 8). As in the transcriptomics analyses, the epigenetic response to M. tuberculosis by AMs strongly implicated host immune response to pathogens and immune-related signaling pathways, which was mainly driven by opening DOCs and regions with increased acetylated H3K27 (Figure 3C and Figure 4C). Although most of the pathways detected using increased DAc were the same for HC subjects, PLWH, and PrEP subjects, the gene count per pathway or GO term was substantially higher in HC subjects (Figure 4C). Examples of genes with DOCs and DAc at the gene transcription start site (TSS) were CXCL10, IFI44L, $A P O B E C 3 A$, and $M X 1$ (Figure 5, A-D).

Covariates do not explain M. tuberculosis-triggered transcriptional and epigenetic differences among groups. The 3 groups differed with respect to several covariates (Table 1). Among the HC group, the proportion of females was higher, and the use of recreational drugs was lower compared with the PLWH and PrEP groups. Subjects in the PrEP group on average were younger compared with the 2 other groups. The duration of ART was longer for PLWH ( mean $=12.12$ years, $S D=7.64$ ) than PrEP subjects (mean $=3$ years, 
A

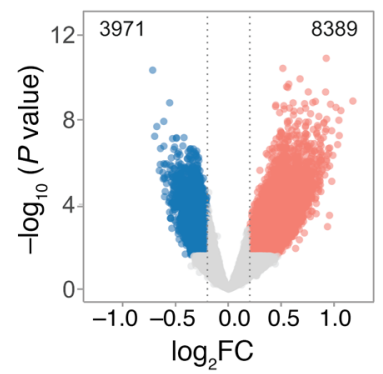

PLWH

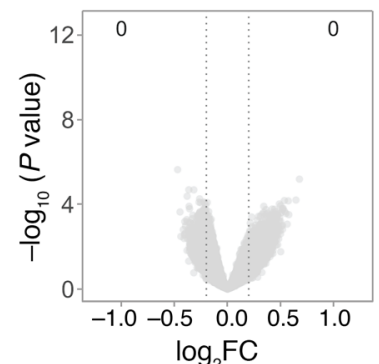

PrEP

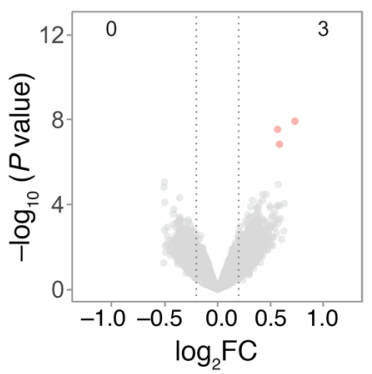

B

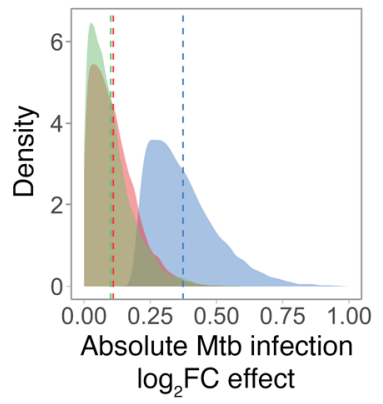

C

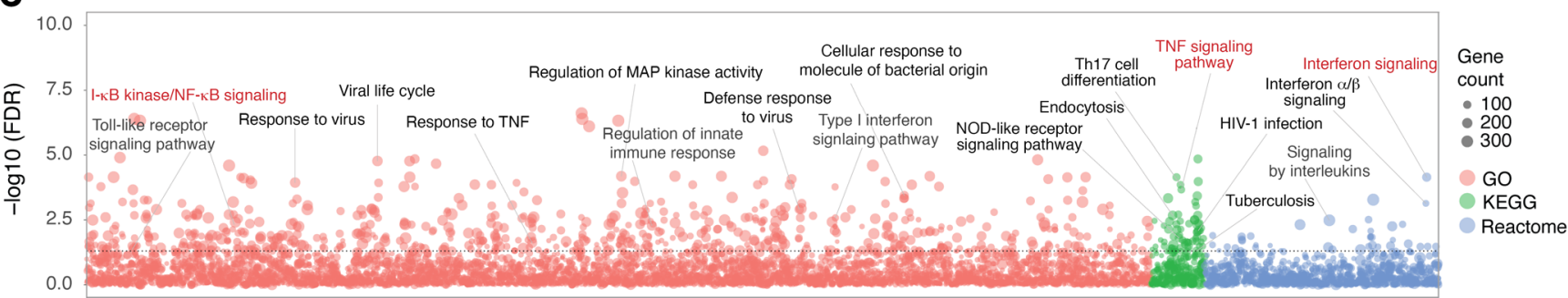

D

I-кB kinase/

NF- $\kappa \mathrm{B}$ signaling (GO BP)
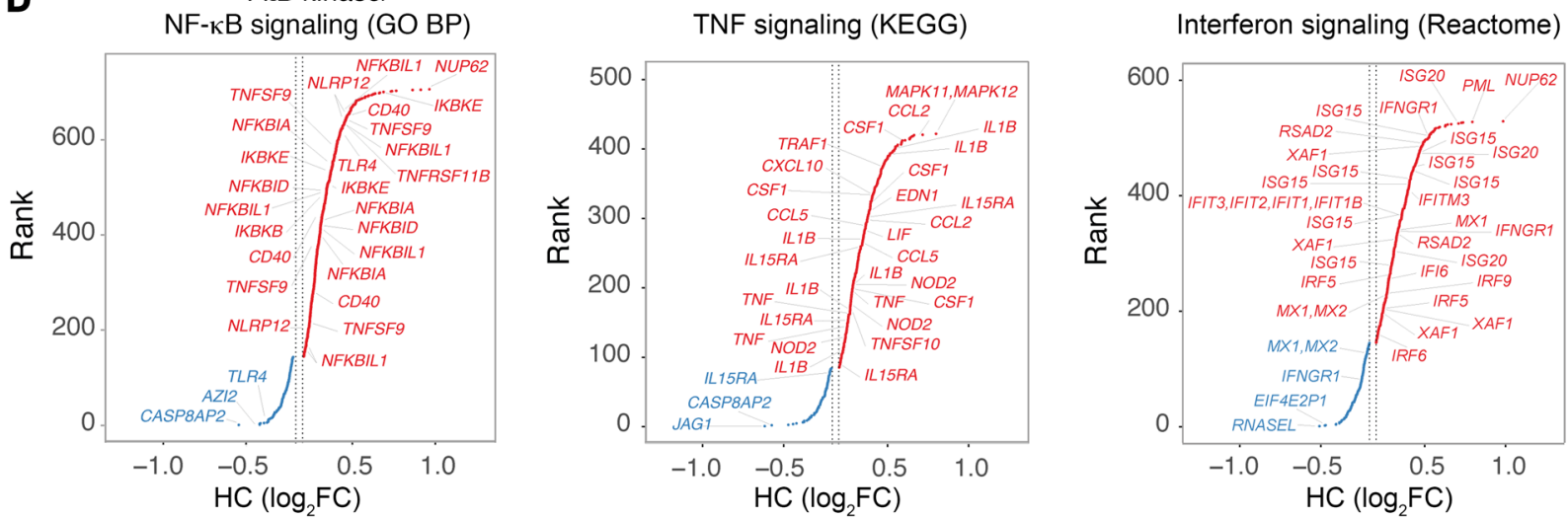

Figure 3. AM chromatin remodeling in response to M. tuberculosis. (A) Significance of chromatin changes is plotted against the magnitude of the change. Significantly open or closed chromatin regions at a FDR of $5 \%$ are represented by red or blue dots, respectively. Counts of DOC regions are given at the top. Vertical dashed lines indicate the minimum $\log _{2} \mathrm{FC}$ of 0.2 on the $x$ axis. For $\mathrm{HC}$ subjects, $23.3 \%$ of the tested regions displayed significant chromatin remodelling in response to $M$. tuberculosis, whereas PLWH and PrEP subjects lacked significant chromatin changes. The raw $P$ value significance cut-off for FDR less than $5 \%$ varies as the FDR procedure is based on the $P$ value distributions that are intrinsic to each tested group. (B) Density of the absolute $\log _{2} \mathrm{FC}$ for 12,360 DOC regions is shown for HC, PrEP, and PLWH groups in blue, green, and red, respectively. Mean log $\mathrm{FC}$ for each group is highlighted by horizontal lines. PrEP and PLWH groups showed strongly impaired chromatin remodelling in response to $\mathrm{M}$. tuberculosis compared with the $\mathrm{HC}$ group. (C) Pathway/GO term enrichment analysis of genes assigned to DOC regions for the HC response to M. tuberculosis. GO biological process (BP), KEGG, and Reactome pathways to which at least 5 genes had been assigned are plotted against the negative $\log _{10}$ FDR. In total, 724 of the 8066 tested terms were significant. Enrichment analysis indicated that $M$. tuberculosis challenge promoted chromatin remodelling in regions assigned to genes belonging to IFN and TNF signaling pathways as well as response to viral and bacterial pathogens. (D) Rank plot of selected terms. DOC regions tagged genes assigned to "I-kappaB kinase/NF-kappaB signaling," "TNF signaling," and "Interferon signaling" pathways. DOCs are plotted according to their log ${ }_{2}$ FC on the $x$ axis and ranked according to the magnitude of change on the $y$ axis. Genes corresponding to opening or closing DOC regions are shown in red and blue, respectively. Horizontal lines indicate the $0.2 \log _{2} \mathrm{FC}$ cut off.

$\mathrm{SD}=1.47)$. All PrEP subjects received Emtricitabine and Tenofovir disoproxil fumarate (TDF), while among PLWH there was a wider spread of ART drug regimens. However, only 4 of 20 subjects had not received TDF at the time of enrollment (Table 1). Most of the intersubject variability was accounted for by the analytical approach employed. Still, given the strong heterogeneity of study subjects, we investigated the impact of smoking, recreational drug use, and age on M. tuberculosis-triggered chromatin and transcriptional responses. We found that smoking and recreational cannabis use did not explain the reduced transcriptional activity or absence of chromatin changes in response to M. tuberculosis among PLWH and PrEP subjects (Figure 2B and Figure 6). Age and duration of ART/PrEP were also unlikely confounders since PLWH and PrEP subjects differed significantly in age $\left(P\right.$ value $_{P L W H \times P r E P}=1.1 \times 10^{-3}$, $P$ value $_{P Y E P \times H C}=$ nonsignificant) and ART duration, yet both groups showed similar reduced transcriptional activity and lack of chromatin changes relative to $\mathrm{HC}$ subjects following challenge with M. tuberculosis (Figure 7, A and B). Moreover, when stratifying HC subjects and PLWH by age, HC subjects of 40 years and older showed pronounced $M$. tuberculosis-triggered chromatin changes 
A

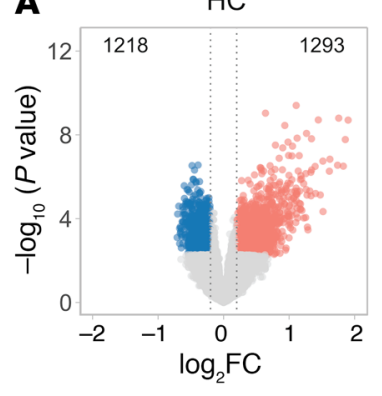

PLWH

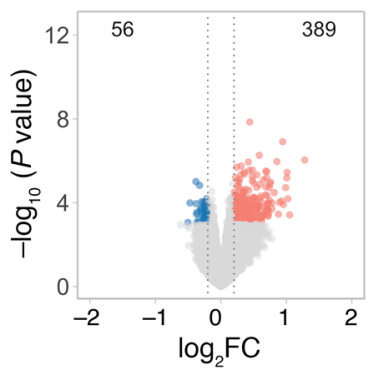

PrEP

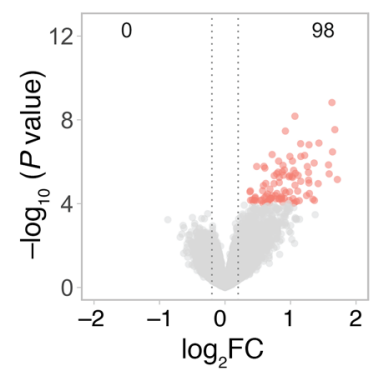

B

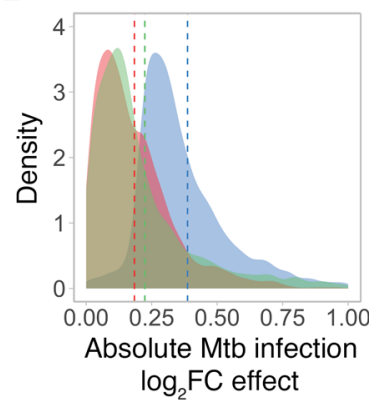

C
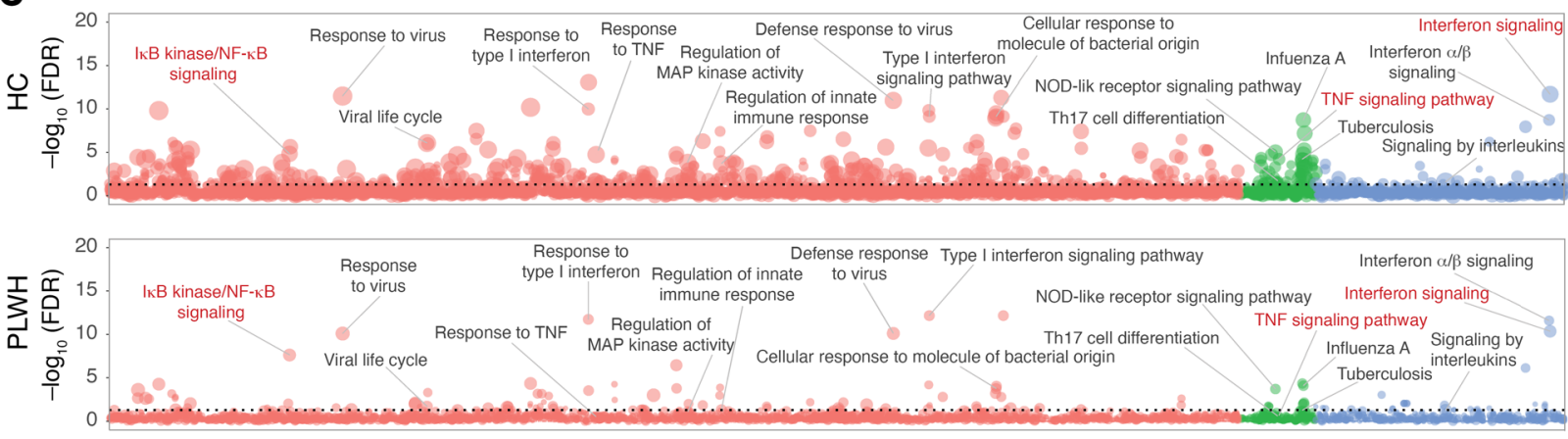

Figure 4. Histone H3K27 acetylation changes in response to M. tuberculosis. (A) Volcano plots for H3K27 acetylation response to M. tuberculosis. Chromatin regions significantly DAc at a FDR less than $5 \%$ and absolute $\log _{2} \mathrm{FC}$ greater than or equal to 0.2 after $M$. tuberculosis challenge are colored, with higher acetylation of H3K27 in red and lower acetylation in blue. The numbers of DAc regions are given at the top. Significant changes in H3K27 acetylation, indicating active enhancers, were observed mostly for the HC group, which encompassed 7 subjects compared with 11 PLWH on antiretroviral therapy and 6 PrEP subjects. Additionally, the magnitude of $\log _{2} \mathrm{FC}$ was higher in $\mathrm{HC}$ subjects compared with PLWH and PrEP subjects. (B) Density of the absolute $\log _{2}$ FC for 2731 DAc regions detected in at least one group is shown for HC, PrEP, and PLWH groups in blue, green, and red, respectively. Mean log ${ }_{2} F C$ for each group is highlighted by vertical lines. PrEP and PLWH groups showed strongly reduced changes in H3K27 acetylation in response to $\mathrm{M}$. tuberculosis compared with the HC group. (C) Enrichment analysis of genes assigned to regions with increased H3K27 acetylation for the HC subjects, PLWH, and PrEP subjects response to M. tuberculosis. GO BP, KEGG, and Reactome pathways with at least 5 assigned genes are plotted against the negative log ${ }_{10}$ FDR. As observed for the chromatin changes, enrichment analysis indicated that in AMs of HC subjects, M. tuberculosis challenge promoted increased acetylation in more regions assigned to genes belonging to IFN and TNF signaling pathways as well as response to viral and bacterial pathogens. Terms highlighted in red are those for which we showed differential M. tuberculosis response between PLWH or PrEP subjects against HC subjects in Figure 2B.

that were not observed for PLWH (Figure 7C). In addition to the covariates listed in Table 1, we also considered possible groupdependent differences in phagocytic activity. Since the yields of AMs were generally not sufficient to conduct independent assessment of phagocytic activity, we used the presence of $M$. tuberculosis reads in ATAC-Seq libraries as proxy and failed to detect any significant group-dependent differences (Figure 7D).

Chromatin accessibility and binding of transcription factors. To link M. tuberculosis-triggered epigenetic changes in AMs from HC subjects with increased gene expression, we evaluated the enrichment of transcription factor (TF) motifs in M. tuberculosis-triggered DOC and DAc regions and found an enrichment of IFN regulatory factor (IRF) motifs in those regions (Figure 8). Next, we compared the average difference in TF footprint depth between $M$. tuberculosis-challenged and nonchallenged AMs to estimate TF activity. Of the 682 TFs obtained from the
JASPAR catalog, 21 had significantly higher activity (increased footprint depth) in DOC regions after M. tuberculosis challenge (Figure 9A). Among these significant TFs were IRF9 (part of the anti-mycobacterial host response) and ZNF684 (a TB biomarker). Of note, $M$. tuberculosis challenge triggered a higher fold change for IRF9 and ZNF684 RNA expression in AMs from HC subjects compared with AMs from PLWH or PrEP subjects (Figure 9B). Moreover, IRF9 and ZNF684 footprints responsive to $M$. tuberculosis challenge were enriched in the TSS of genes that are part of the IFN signaling pathway $\left(P=8.9 \times 10^{-6}\right.$ and $P=$ 0.03 , respectively; Figure 9 C). Unexpectedly, we also observed an enrichment of IRF9 active footprints in the TSS of genes of the TNF signaling pathway $(P=0.01$; Figure $9 C)$. ZNF684 active footprints were observed in the core promoter of TNF after $M$. tuberculosis challenge, providing a direct link to a major proinflammatory cytokine (Figure 9D). Moreover, in support of a coor- 
A

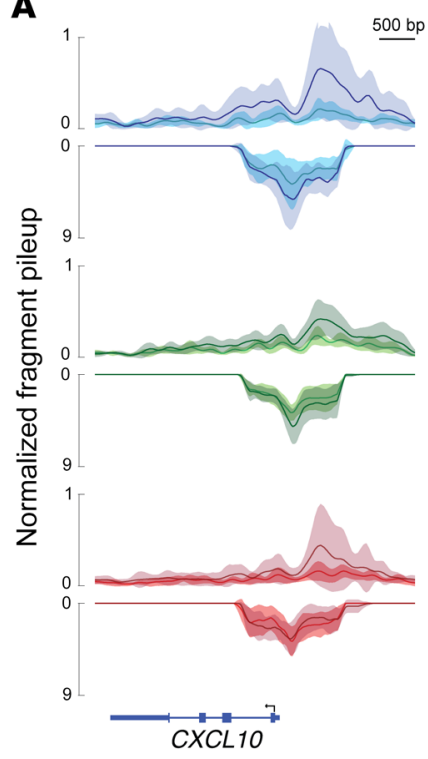

B

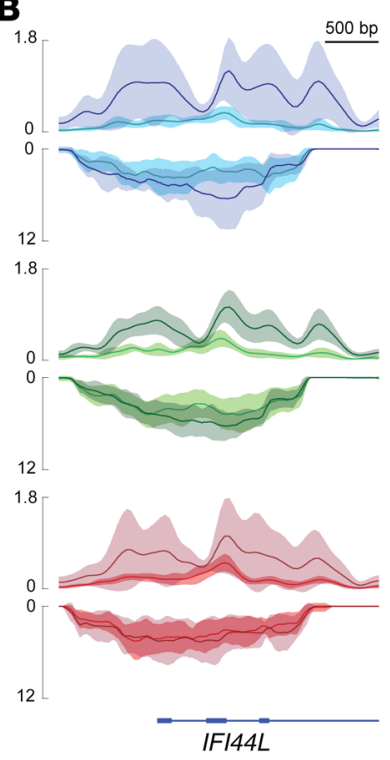

C
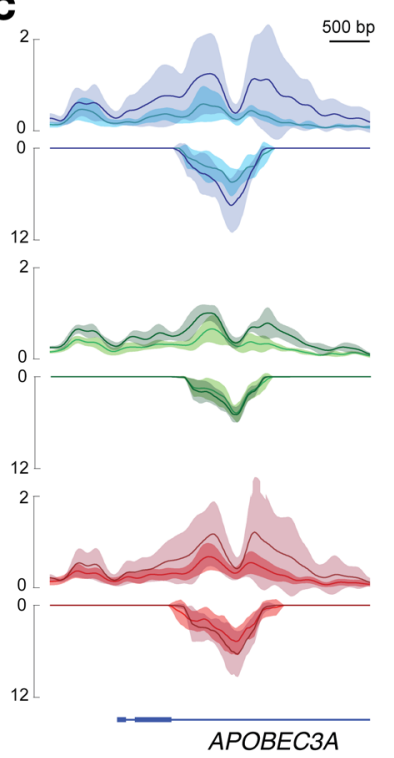

D

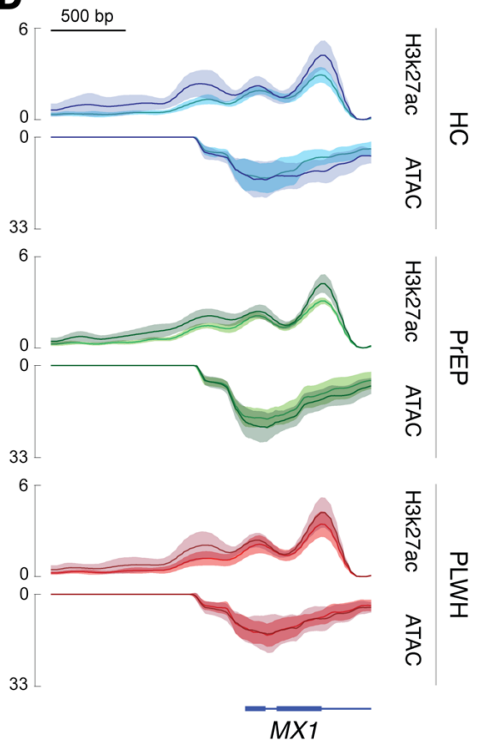

Figure 5. Changes in chromatin accessibility and H3K27 acetylation of DEGs with high $\log _{2} \mathrm{FC}$ in response to $M$. tuberculosis. Chromatin accessibility and H3K27 acetylation for HC subjects, PrEP subjects, and PLWH while receiving antiretroviral treatment are depicted for the TSS of 4 DEGs-CXCL10 (A), IFI44L (B), $A P O B E C 3 A(\mathbf{C})$, and $M X 1$ (D)-that are among the genes displaying the highest $\log _{2} \mathrm{FC}$ transcriptional changes in response to $M$. tuberculosis. The mean normalized fragment pileup for H3K27 acetylation (top) and chromatin accessibility (bottom) are plotted on the $y$ axis for HC, PrEP, and PLWH groups and colored in blue, green, and red, respectively. The lines indicate the mean H3K27 acetylation (top) and chromatin accessibility (bottom) for each condition in the group with a light shade representing noninfected AMs and a darker shade representing the $M$. tuberculosis-challenged AMs. The standard deviation of the mean is shown by the corresponding shades for each condition. A complementary effect was observed with DOC region in response to $M$. tuberculosis being flanked by increased H3K27 acetylation for the genes displayed.

dinated effect of these 2 TFs, an active footprint for ZNF684 was observed in the promoter region of IRF9 (Figure 9D).

To further assess the link between $M$. tuberculosis-dependent TF binding and RNA expression, we evaluated whether the 135 expressed genes with active IRF9 footprint at the TSS were differentially expressed in $\mathrm{HC}$ subjects following M. tuberculosis challenge. We observed that 65 of the 135 genes were DEGs ( 57 upregulated and 8 downregulated) following $M$. tuberculosis challenge $\left(P=7.9 \times 10^{-23}\right.$; Supplemental Tables 9 and 10$)$. AMs from PLWH and PrEP subjects had lower $\log _{2} \mathrm{FC}$ in response to $M$. tuberculosis for all 65 DEGs (Supplemental Table 10). Of the 57 upregulated DEGs with IRF9 TSS footprint, 17 were part of the IFN or TNF pathways, including NOD2 and ISG15 (Figure 9D). Collectively, these data showed that the epigenetic response observed in AMs from HC subjects promoted the binding of key TFs required for IFN and TNF signaling. The locked state of chromatin in AMs from PLWH and PrEP subjects was therefore likely to account, at least in part, for the reduced transcriptional activity of those cells in response to $M$. tuberculosis.

\section{Discussion}

There is a scarcity of knowledge about the early fate of inhaled $M$. tuberculosis bacilli in the human lung. Infection of human hosts with $M$. tuberculosis is inferred from detectable anti-M. tuberculosis immunity through either the in vivo tuberculin skin test (TST) or ex vivo IFN- $\gamma$ release assays (IGRAs) (17). However, these tests reflect $\mathrm{T}$ cell immunity that is being generated in the lymph nodes, and not in the lung, several weeks after the initial uptake of $M$. tuberculosis (18). Consequently, much of our knowledge about the
M. tuberculosis-host interplay from inhalation to the development of acquired $\mathrm{T}$ cell immunity has been derived from observations in animal models. In the mouse, $M$. tuberculosis bacilli in the lung alveoli are taken up by AMs (7). Infected AMs function as a niche for $M$. tuberculosis replication prior to the migration of M. tuberculosis-infected AMs to the lung interstitium where M. tuberculosis bacilli are transferred to inflammatory macrophages that facilitate the induction of $M$. tuberculosis-specific T cell immunity (7). The increased permissiveness of AMs for M. tuberculosis in the mouse has been confirmed in a number of independent studies and appears to reflect the metabolic state of the cells $(19,20)$.

It is not known how well this scenario is replicated in the human lung. One critical difference between the mouse model and humans is the ability of a subgroup of M. tuberculosis-exposed persons to avoid the development of classical T cell immunity and TB disease even in the presence of long-lasting M. tuberculosis exposure (9). Recently, the phenotype of strong resistance to immune conversion of persons living in high $M$. tuberculosis exposure settings has been confirmed for HIV-infected but immune-reconstituted people (21). While the underlying mechanisms that preclude the development of classical $\mathrm{T}$ cell immunity are unknown, initial results implicate both nonclassical T cells and B cells $(21,22)$. How and where AMs interact with these cells of the acquired immunity arm is not known. We also do not know how the strong persistent exposure of human AMs to environmental stimuli and pollutants affects AM physiology and anti-mycobacterial activity. An important influence of the environment on AM physiology is consistent with the pronounced interindividual differences observed in our study. Nevertheless, in our experiments all AMs displayed an 

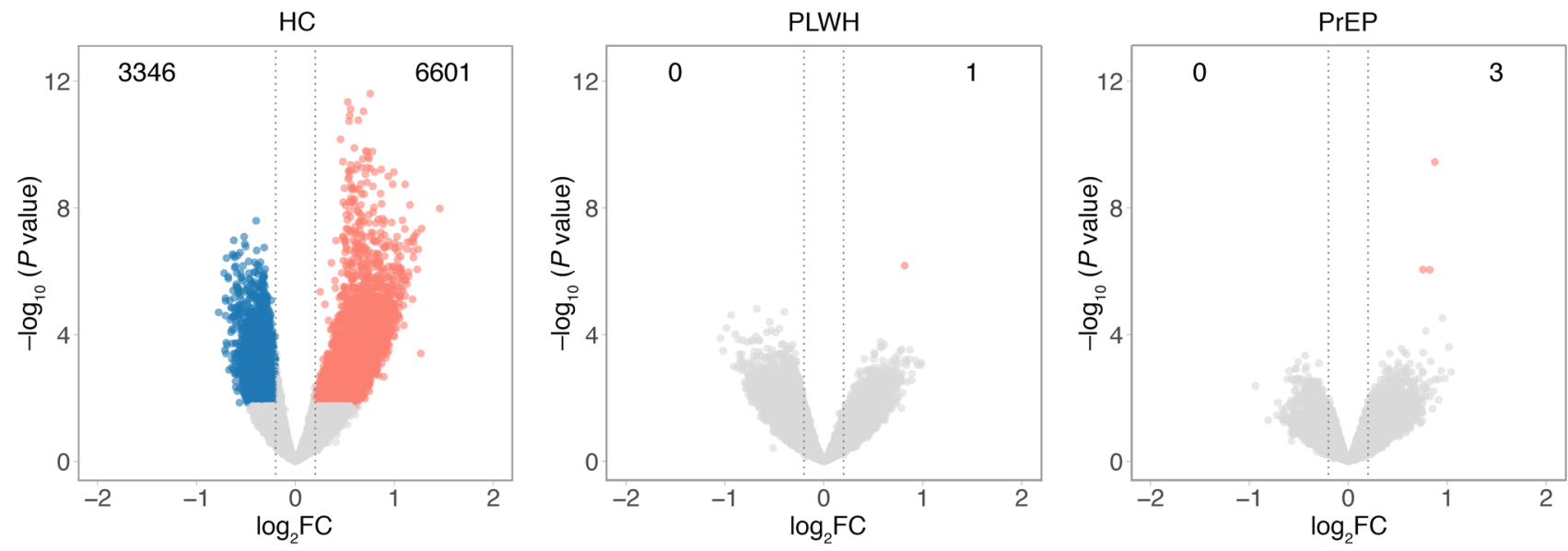

Figure 6. No significant impact of recreational cannabis use and smoking on M. tuberculosis-triggered epigenetic changes in AMs. Volcano plots for chromatin accessibility changes by AMs in response to $M$. tuberculosis after removing subjects who smoked cigarettes, cannabis, or both. Included in this analysis were $10 \mathrm{HC}$ subjects, $7 \mathrm{PLWH}$, and 5 PrEP subjects. Significantly open or closed chromatin regions at a FDR of 5\% after M. tuberculosis challenge are represented by dots marked in red or blue, respectively. The DOC regions are given at the top left and right corners. Dashed lines indicate the $5 \%$ FDR on the $y$ axis and the minimum $\log _{2} F C$ of 0.2 on the $x$ axis.

innate anti-mycobacterial immune response to M. tuberculosis that was dominated by IFN and TNF signaling, suggesting that AMs are directly involved in the host defense against $M$. tuberculosis. However, this anti-M. tuberculosis response was significantly weaker in AMs from PLWH and PrEP subjects.

The impetus for our experiments was the well-known observation that PLWH are at increased risk to become infected with M. tuberculosis and to develop clinical TB disease $(4-6,23)$. This increased susceptibility is still present in PLWH who have been immune-reconstituted with ART $(2,24)$. A detrimental effect of HIV on AM function has been shown in pre-ART patients, including on their response to M. tuberculosis (25). In our study we found no evidence for reduced phagocytic uptake of $M$. tuberculosis by AMs across phenotypic groups, arguing against a confounding effect of HIV or ART on phagocytic activity. This is consistent with previous reports that AMs obtained from PLWH showed no defect in phagocytosis of Salmonella Typhimurium compared with HIV-negative controls (26). AMs from PLWH on long-term ART displayed a decreased ability to kill Streptococcus pneumoniae due to their HIV gp120-mediated reduced AM apoptotic activity (27, 28). Consistent with this finding, reduced AM phagocytic activity of Staphylococcus aureus in a sample of PLWH/ART subjects was associated with detectable proviral DNA (29). In our sample we found that 6 of the 7 tested PLWH subjects had undetectable proviral counts, arguing against a role of provirus in our sample (30). Moreover, the differences in the transcriptional response to M. tuberculosis between the PrEP and PLWH groups were less pronounced as compared with the differences of both groups to HC participants, which argues against HIV being the main cause of the blunted anti-M. tuberculosis response by AMs.

The differences in the transcriptional response between the phenotype groups were graded and quantitative. However, the changes in M. tuberculosis-triggered chromatin structure of AMs were sharply divided between PLWH and PrEP subjects compared with controls. Indeed, AMs from both PLWH receiving ART and PrEP subjects displayed a near total lack of chromatin changes in response to $M$. tuberculosis. This suggested an unknown effect on chromatin of the drugs used in the PrEP and ART regimen. Yet, how drugs might impact the AM epigenetic responsiveness and the transcriptional response to M. tuberculosis is not clear. While the observed increased TF binding in DOC/DAc regions is consistent with the idea of the chromatin lock-down being the cause of the reduced transcriptional response, this causality remains to be more stringently established. Irrespective of the precise mechanisms, the blunted ability of AMs to mount a response to an infectious insult may contribute to the known increased vulnerability to pulmonary disease of PLWH. For example, the prevalence of bacterial pneumonias and chronic obstructive pulmonary disease (COPD) are increased in PLWH/ART $(31,32)$. PLWH/ART also experience an increased risk of sepsis with poor outcome (33). However, a more direct link of our findings can be made with TB, for which PLWH on long-term ART who have been immune reconstituted remain at increased risk $(2,24)$. Since our data suggest that the blunted AM response to $M$. tuberculosis is predominantly mediated by the anti-viral drugs and not the virus, use of PrEP in settings of high TB transmission might require consideration of the risk of TB versus protection from HIV. Our results also need to be seen in the context of the ongoing COVID-19 pandemic. Several large studies have consistently shown that PLWH are at increased risk of severe disease and death resulting from SARS-CoV-2 infection $(34,35)$. While the initial pulmonary host cells of SARS-CoV-2 are alveolar type 1 and type 2 cells, AMs are a critical part of the pulmonary host response (36). A reduced interferon response by AMs, as observed for PLWH and PrEP subjects in our study, has been associated with poor outcomes and death from COVID-19 (36). Our results for infection with M. tuberculosis suggest that the interplay of reduced host responsiveness, anti-retroviral therapy, and COVID-19 outcome deserves further study. Nevertheless, a limitation of the present study was our inability to enroll patients who had been newly diagnosed with HIV before the onset of ART. The dissection of the relative impact of HIV and PrEP on host immune response will require additional experiments employing a different study design. 
A

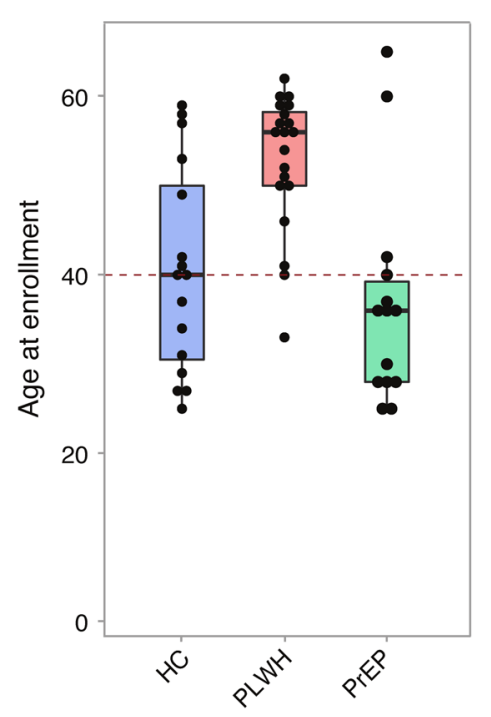

C

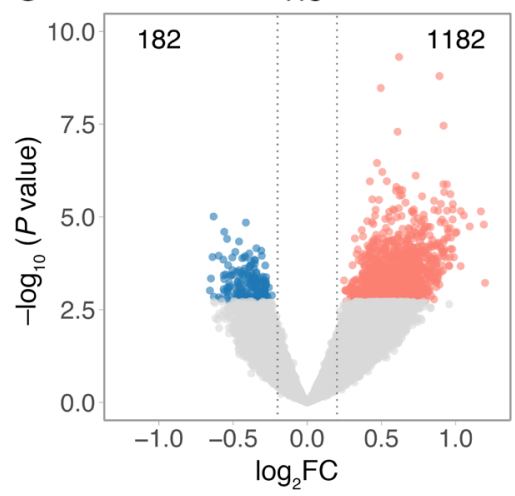

B
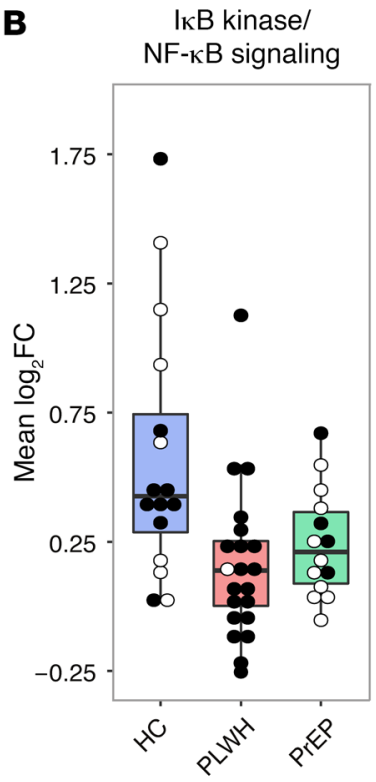
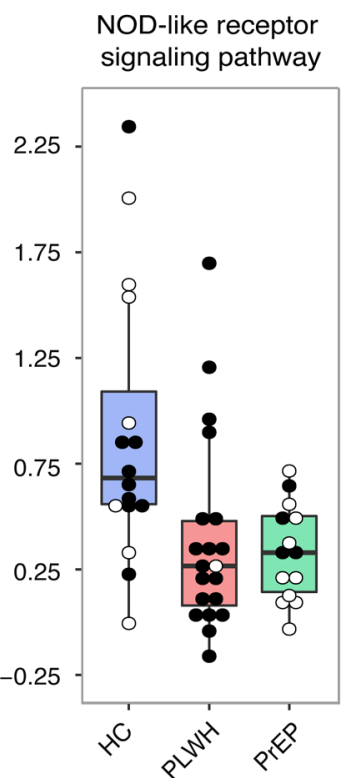

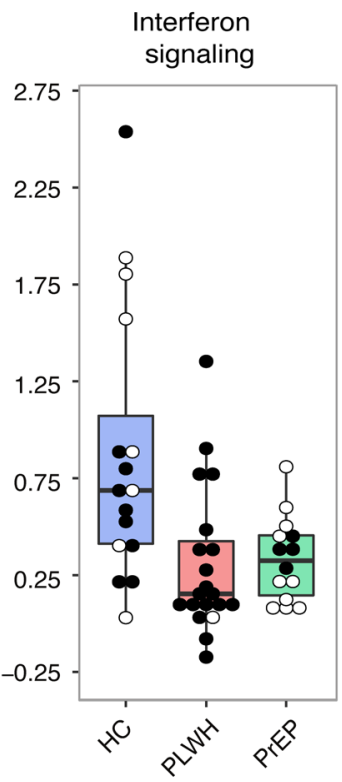

Interferon signaling

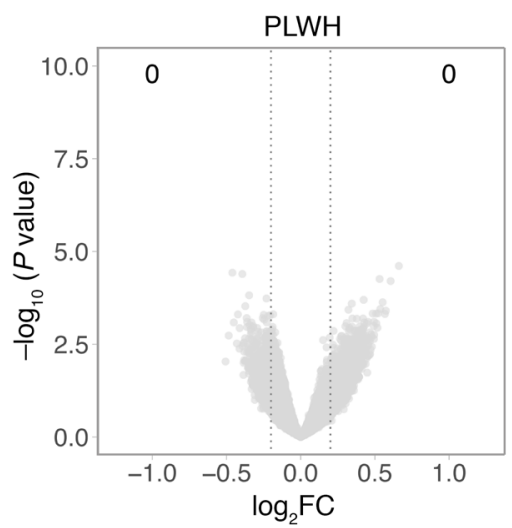

D

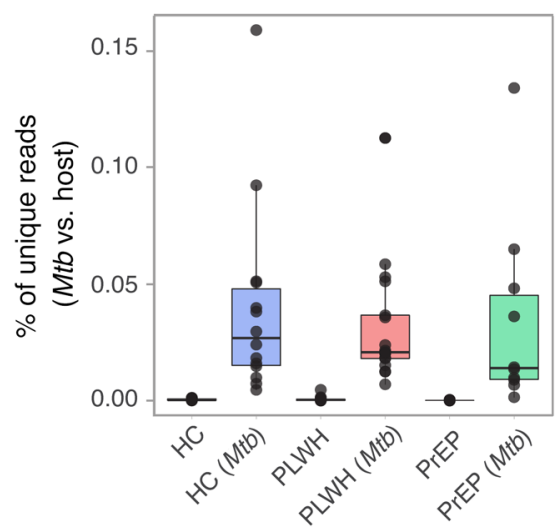

Figure 7. Impact of age and M. tuberculosis uptake on transcriptional and epigenetic changes. (A) Boxplot showing the distribution of age at enrollment across the 3 studied groups. Subjects of the PLWH group were significantly older than the subjects of the HC and PrEP groups. (B) Boxplot for 3 selected pathway/GO terms for genes with significant differential M. tuberculosis response between PLWH or PrEP subjects against HC subjects ( $n=302$ ). For each term, the $y$ axis displays the mean $\log _{2} F C$ and dots represent per-subject mean value for pooled DEGs from the 3 groups. White dots represent mean log ${ }_{2} F C$ for subjects younger than 40 years at enrollment, whereas black dots depict subjects 40 years of age or older. (C) Volcano plots for chromatin accessibility changes stratified by age for subjects of the HC and PLWH groups. Subjects tested were at least 40 years old at enrollment. In this age class, the HC, PLWH, and PrEP groups encompassed 9, 15, and 3 subjects, respectively. Due to low subject count, PrEP subjects were excluded from this stratified analysis. Significantly open or closed chromatin regions at a FDR of $5 \%$ after $M$. tuberculosis challenge are represented by dots marked in red or blue, respectively. The counts of DOC regions are given at the top left and right corners. Vertical dashed lines indicate the $\log _{2} \mathrm{FC}$ of 0.2 cut-off on the $x$ axis. The raw $P$ value cut-off that is controlled under FDR less than $5 \%$ varies, as the FDR procedure is based on the $P$-value distributions that are intrinsic to each tested group. (D) Boxplot of distribution for M. tuberculosis-aligned reads in ATAC-Seq experiments. The $y$ axis indicates the proportion of total unique pairedend reads aligned to the $M$. tuberculosis H37rv genome relative to the human genome hg38. Unique alignments to the M. tuberculosis genome quantify DNA traces of phagocyted/lysed mycobacteria. In the absence of $M$. tuberculosis challenge, little to no alignment was observed to $M$. tuberculosis genome. In the challenge condition, the 3 groups (HC, PLWH, and PrEP) presented a range of proportions of $M$. tuberculosis unique reads. No statistical significance was observed between the proportion of $M$. tuberculosis reads between the phenotype groups.

Emtricitabine and Tenofovir, the 2 active components of Truvada, are nucleoside reverse transcriptase inhibitors (NRTIs). NRTI drugs are known to interact with mitochondrial DNA polymerase, an interaction that may underlie some of the adverse effects of Truvada (37). The daily use of Truvada has been associated with mild adverse effects in some patients and may impact kidney or liver function in a small number of patients $(38,39)$. On the molecular level, Truvada has been linked to mitochondrial dysfunction, increased oxidative stress, and cellular senescence, suggesting a possible role for trained immunity $(40,41)$. Trained immunity in the context of $\mathrm{TB}$ is characterized by epigenetic imprinting and rewiring of cellular metabolism, and its circumvention by $M$. tuberculosis in hematopoietic stem cells (42). Interestingly, the concept of trained immunity also applies to long-lived tissue macrophages. Studies in the mouse model have demonstrated that viral respiratory infections can imprint efficient and 


\begin{tabular}{|c|c|c|c|c|c|c|}
\hline Test & TFs & Motif & $P$ value & FDR & $\begin{array}{l}\text { Target regions } \\
\text { with motif (\%) }\end{array}$ & $\begin{array}{c}\text { Background regions } \\
\text { with motif }(\%)\end{array}$ \\
\hline Open & ISRE & $A G$ & $1.0 \times 10^{-12}$ & $<1.0^{-5}$ & $288(4.44 \%)$ & $1131(2.85 \%)$ \\
\hline Open & IRF2 & & $1.0 \times 10^{-7}$ & $<1.0^{-5}$ & $429(6.62 \%)$ & $2032(5.12 \%)$ \\
\hline Open & IRF1 & GAAAEIGAA & $1.0 \times 10^{-5}$ & $3.00 \times 10^{-4}$ & $510(7.87 \%)$ & $2544(6.41 \%)$ \\
\hline Open & bHLHE40 & & $1.0 \times 10^{-5}$ & $6.00 \times 10^{-4}$ & $1038(16.01 \%)$ & $5587(14.07 \%)$ \\
\hline Open & NFkB-p50,p52 & $\mathrm{GO}$ & $1.0 \times 10^{-4}$ & $1.10 \times 10^{-3}$ & $706(10.89 \%)$ & $3702(9.33 \%)$ \\
\hline Open & Egr2 & 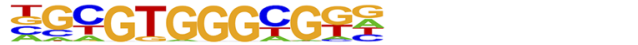 & $1.0 \times 10^{-4}$ & $1.60 \times 10^{-3}$ & $1061(16.37 \%)$ & $5773(14.54 \%)$ \\
\hline Open & bZIP:IRF & 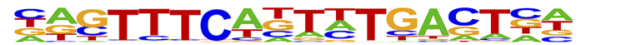 & $1.0 \times 10^{-3}$ & $3.12 \times 10^{-2}$ & $887(13.68 \%)$ & $4888(12.31 \%)$ \\
\hline Open & Hoxb4 & TGAITAATECCC & $1.0 \times 10^{-3}$ & $3.26 \times 10^{-2}$ & $396(6.11 \%)$ & $2059(5.19 \%)$ \\
\hline Open & c-Myc & & $1.0 \times 10^{-3}$ & $3.91 \times 10^{-2}$ & $1666(25.70 \%)$ & $9530(24.01 \%)$ \\
\hline Open & Slug & 옴출 & $1.0 \times 10^{-3}$ & $3.91 \times 10^{-2}$ & $1895(29.23 \%)$ & $10909(27.48 \%)$ \\
\hline Open & Oct4:Sox17 & A스르를 & $1.0 \times 10^{-3}$ & $3.91 \times 10^{-2}$ & $252(3.89 \%)$ & $1262(3.18 \%)$ \\
\hline Open & REST-NRSF & STSCTGA & $1.0 \times 10^{-2}$ & $4.17 \times 10^{-2}$ & $39(0.60 \%)$ & $140(0.35 \%)$ \\
\hline More acetylated & ISRE & $A G$ & $1.0 \times 10^{-121}$ & $<1.0^{-5}$ & $299(28.56 \%)$ & $2131(5.66 \%)$ \\
\hline More acetylated & IRF2 & RAAACCAAAACC & $1.0 \times 10^{-108}$ & $<1.0^{-5}$ & $352(33.62 \%)$ & $3379(8.98 \%)$ \\
\hline More acetylated & IRF1 & GAAACIGAAAEI & $1.0 \times 10^{-106}$ & $<1.0^{-5}$ & $407(38.87 \%)$ & 4578 (12.17\%) \\
\hline More acetylated & IRF3 & 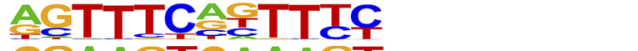 & $1.0 \times 10^{-92}$ & $<1.0^{-5}$ & $580(55.40 \%)$ & 9596 (25.50\%) \\
\hline More acetylated & IRF8 & GEAAEIGAAAEI & $1.0 \times 10^{-79}$ & $<1.0^{-5}$ & $558(53.30 \%)$ & $9689(25.75 \%)$ \\
\hline More acetylated & PU.1:IRF8 & GGAAGIGAAAEI & $1.0 \times 10^{-36}$ & $<1.0^{-5}$ & $351(33.52 \%)$ & $6497(17.27 \%)$ \\
\hline More acetylated & T1ISRE & АCTICGTII & $1.0 \times 10^{-19}$ & $<1.0^{-5}$ & $52(4.97 \%)$ & $385(1.02 \%)$ \\
\hline More acetylated & IRF4 & & $1.0 \times 10^{-18}$ & $<1.0^{-5}$ & $418(39.92 \%)$ & $10188(27.08 \%)$ \\
\hline More acetylated & bZIP:IRF & & $1.0 \times 10^{-7}$ & $<1.0^{-5}$ & $376(35.91 \%)$ & $10538(28.01 \%)$ \\
\hline More acetylated & PRDM1 & 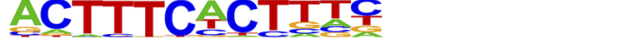 & $1.0 \times 10^{-7}$ & $<1.0^{-5}$ & $459(43.84 \%)$ & $13444(35.73 \%)$ \\
\hline More acetylated & PU.1-IRF & СGGAAGTGAАAC & $1.0 \times 10^{-5}$ & $1.00 \times 10^{-4}$ & $779(74.40 \%)$ & $25590(68.01 \%)$ \\
\hline
\end{tabular}

Figure 8. Motif enrichment analysis for ATAC-Seq and H3K27ac. Motifs enriched in open or more acetylated chromatin of HC subjects upon M. tuberculosis challenge are shown as a table. The tested conditions with corresponding TFs binding to the enriched motifs are shown in the first 3 columns. In the last 4 columns, the $P$ value and FDR are given for the enrichment of motifs in targeted regions versus the background. Using HOMER default parameters, 6486 of the 8389 more accessible regions (ATAC) and 1048 of the 1293 more acetylated regions (H3k27ac) for the HC response to M. tuberculosis passed quality control and were considered target regions. The background consisted of regions with FDR greater than $5 \%$ for each comparison. Using HOMER default parameters, 39,695 of the 44,651 background regions (ATAC) and 55,466 of the 64,564 background regions (H3k27ac) passed quality control. The percentage relative to the total number of regions that passed QC in either targeted regions (open or more acetylated) or background are indicated in the last 2 columns.

long-lasting anti-bacterial trained immunity on AMs. Anti-bacterial immunity by AMs is imprinted by IFN- $\gamma$-secreting $\mathrm{CD}^{+} \mathrm{T}$ cells and mediated via a vigorous chemokine response and neutrophilia in the lung (43). In PLWH on ART, in pulmonary CD8 $8^{+} \mathrm{T}$ cells have been shown to have a functional impairment, which is consistent with a role of disrupted trained immunity of AMs in our experiments (44). Finally, no data are presently available that link PrEP with risk of $\mathrm{TB}$ or other respiratory diseases. On the other hand, several studies reported an increase of sexually transmitted infections, often asymptomatic, among PrEP users (45). While this increase is commonly ascribed to increased risk-taking behavior among some PrEP users, in the light of our findings alternative contributing factors should be considered in future studies.

\section{Methods}

Subjects. HIV-negative participants represented a control group $(n=$ 23) and a high-risk group on preventive therapy (PrEP, $n=14$ ). HIV positive participants ( $\mathrm{PLWH}, n=27$ ) were receiving ART to suppress peripheral viral load for at least 3 years and without any active respiratory symptoms or infections. Exclusion criteria included past history of TB, pregnancy, chronic cardiovascular or active pulmonary infec- tion, and immunosuppressive medication. All participants were tested with QuantiFERON Gold (catalog 0594-2010/T0590-0301; Qiagen) and one subject each of the HC and PrEP groups tested positive. Since exclusion of these 2 subjects had no significant impact on reported results, we left them included in the present presentation of the data. Information on participant characteristics and antiretroviral drugs was extracted from the clinical database and participant electronic records. Of these enrolled subjects, we excluded $7 \mathrm{HC}$ subjects and 7 PLWH from the analyses due to low yield of BAL cells, or inability to pass quality control for sample or library preparation.

Bronchoalveolar lavage and sample processing. Bronchoscopies were performed at the Centre for Innovative Medicine of the Research Institute of the McGill University Health Centre following the guidelines of the American Thoracic Society (46). Briefly, standard flexible bronchoscopy of the middle lobe was conducted under local anesthesia with lidocaine with additional conscious sedation with intravenous midazolam. A total of $200 \mathrm{~mL}$ sterile saline instilled in $50 \mathrm{~mL}$ volumes resulted in an average return of $115 \mathrm{~mL}$ (range of $50-150 \mathrm{~mL}$ ) of bronchoalveolar lavage (BAL) fluid. BAL fluid in nonadherent tubes was stored on ice for a maximum of 30 minutes prior to the isolation of AMs. All BAL fluids were strained through a 100 $\mu \mathrm{m}$ filter to remove any mucus and cell clumps. Cytology assessment 

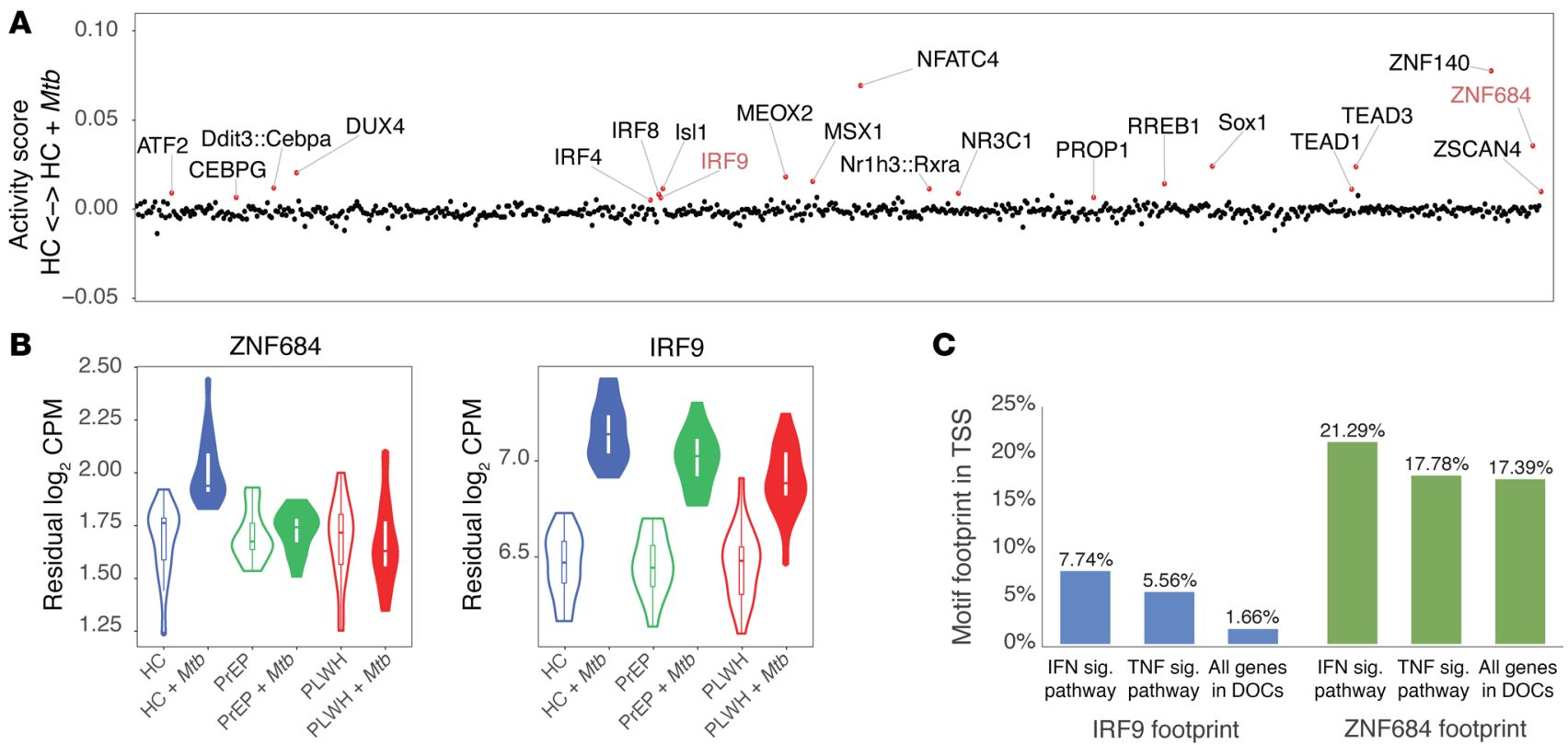

D Chr6: 31,575,000-31,576,600
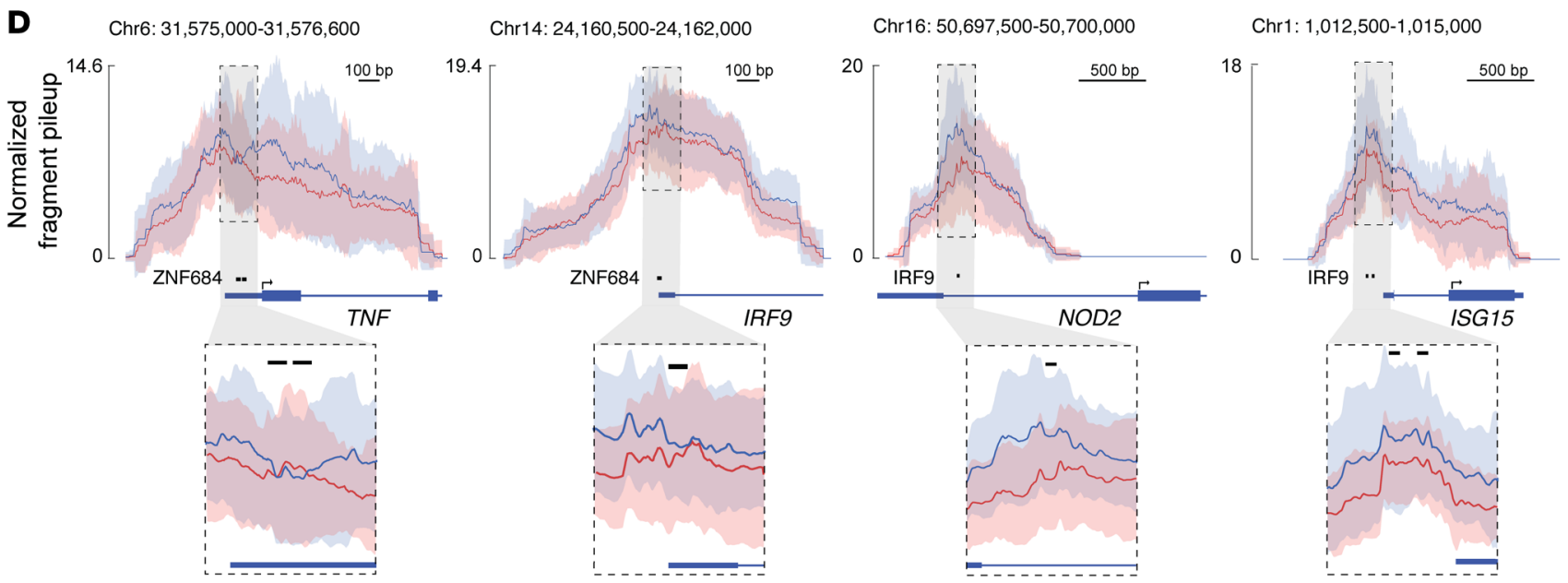

Figure 9. TF footprint activity in DOC regions. (A) Activity scores denoting differences in depth of TF footprints between HC subjects and HC subjects challenged with $M$. tuberculosis AMs are plotted on the $y$ axis for 682 TFs from the JASPAR catalog showing a minimum of 50 footprints in DOC regions. A positive activity score indicates that footprints for a TF are more pronounced in $\mathrm{HC}$ after $M$. tuberculosis infection. Twenty-one TFs with significant activity score at FDR less than $5 \%$ are displayed as red dots. (B) mRNA expression levels for the transcription factors IRF9 and ZNF684 are shown as violin plots. The residual $\log _{2}$ copy per million (CPM) read, after removing interindividual variability, is plotted for each group: HC subjects, PrEP subjects, and PLWH receiving antiretroviral treatments and condition ( $M$. tuberculosis-challenged or not). (C) Percentage of genes in the TNF or IFN pathways that have IRF9 or ZNF684 footprints in their TSS ( $\pm 5 \mathrm{~kb}$ from the promoter) was compared with all genes with TSS in DOC regions. As expected, we observed that IRF9 footprints are more frequent in genes assigned to Reactome's IFN signaling pathway compared with random TSS in DOCs. Surprisingly, IRF9 footprints were also more frequent than by chance in KEGG's TNF pathway. (D) ZNF684 and IRF9 footprints in selected IFN and TNF genes. The mean normalized fragment pileup is plotted on the $y$ axis as red and blue lines for noninfected and M. tuberculosis-challenged AMs from HC subjects, respectively. The standard deviation of the mean is shown by shades for each condition. The location of the motif assigned to the footprint is shown by a black bar. Pronounced footprints for ZNF684 were in the core promoter of TNF and the promoter of IRF9. Motifs for IRF9 were detected in footprints located close to the promoter of the NOD2 and ISG15 genes. TF footprints are detected by a depletion in sequencing depth between 2 high coverage flanking regions. The bottom panel zooms in at footprints located over TF motifs with higher activity score in HC subjects and located in the promoter region of top DEGs in response to M. tuberculosis.

of BAL samples indicated that AMs represented more than $90 \%$ of all recovered mononuclear cells.

Alveolar macrophage preparation. AMs were isolated by adhesion. Collected BAL cells were spun for 10 minutes at $300 \mathrm{~g}, 10^{\circ} \mathrm{C}$, and washed twice in RPMI-1640 with L-glutamine (Wisent), containing $2 \%$ human serum (heat-inactivated $\mathrm{AB}+$ off the clot, Wisent), $1 \%$ penicillin/streptomycin (Gibco), 10 mM HEPES (Gibco), 1\% nonessential amino acids (Gibco), and $1.75 \mathrm{ng} / \mathrm{mL}$ Amphotericin B (Wisent). After the cell count, $1.5 \times 10^{6} \mathrm{AMs}$ were seeded per well of a 6-well plate (Falcon) in RPMI-1640 supplemented with $10 \%$ human serum and incubated for $1.5-2$ hours at $37^{\circ} \mathrm{C}, 5 \% \mathrm{CO}_{2}$, and $95 \%$ relative humidity. Nonadherent cells were removed by thorough washings with prewarmed $\left(37^{\circ} \mathrm{C}\right)$ RPMI-1640 supplemented with $2 \%$ human serum. Washed adherent AMs were then infected with freshly prepared M. tuberculosis. 
An estimate for the number of adherent AMs was derived by subtracting the number of detached from the number of seeded cells.

Mycobacterial cultures and macrophage challenge in vitro. Virulent M. tuberculosis strain H37Rv was grown in a liquid culture of Middlebrook 7H9 medium (Difco, catalog 295939) containing 0.2\% glycerol (Thermo Fisher Scientific), 0.05\% Tween-80 (Sigma-Aldrich), and $10 \%$ albumin-dextrose-catalase (ADC) (Thermo Fisher Scientific) at $37^{\circ} \mathrm{C}$ in rolling incubators. Bacteria were grown to log phase, as determined by an optical density of 0.3 to 0.8 at $600 \mathrm{~nm}$, prior to inoculum preparation. Further, bacterial cultures were spun for 15 minutes at $3500 \mathrm{~g}$, resuspended in RPMI-1640, and dislodged with a $22 \mathrm{G}$ needle. Cell suspensions were filtered through $5 \mu \mathrm{m}$ filters (Millipore) to ensure single mycobacteria suspensions for the challenge experiments. Bacterial counts of inocula were done using disposable Neubauer hemocytometer (C-Chip, INCYTO). Bacterial loads were confirmed by CFU counts by plating serial dilution of inoculum in 7H9 growth medium on Middlebrook 7H10 agar (Becton Dickinson, catalog 254520) plates containing 0.5\% glycerol (Thermo Fisher Scientific) and 10\% oleic acid-ADC (OADC, Becton Dickinson, catalog 211886). Colonies were counted 3 to 4 weeks after plating. AMs were infected at a MOI of 5:1 for 18 to 20 hours at $37^{\circ} \mathrm{C}, 5 \% \mathrm{CO}_{2}$, and $95 \%$ relative humidity.

Preparation of RNA-Seq and ATAC-Seq libraries. For downstream RNA and ATAC experiments, AMs were rinsed with warm PBS to remove damaged cells and debris before further processing. For RNA, cells were lysed with Trizol and kept at $-80^{\circ} \mathrm{C}$ until extraction. For RNA isolation the miRNeasy kit (Qiagen) was used and RNA integrity (RIN) was assessed with the Agilent 2100 Bioanalyzer (Agilent Technologies). Only samples with RIN greater than 8 were selected for library preparation using TruSeq RNA Library Preparation Kit v2, Set A (Illumina).

For chromatin accessibility using the ATAC-Seq method $(47,48)$, AMs were lifted with prewarmed CellStripper solution (Corning) and gentle pipetting. Viability was assessed with trypan blue staining, and only samples with viability greater than $85 \%$ were used. We followed the protocol by Buenrostro et al. (48). Briefly, 50,000 cells were used for generating challenged and nonchallenged libraries. AMs were permeabilized with lysis buffer containing 0.05\% IGEPAL (Sigma). Nuclei were incubated with Tn5 transposase (Illumina) and additional ATAC-Seq libraries were amplified for a total of 5 to 10 PCR cycles. Barcoded and multiplexed RNA- or ATAC-Seq libraries were sequenced on an Illumina HiSeq4000 sequencer at Genome Quebec, Montreal, Canada.

ChIP-Seq library preparation and sequencing. Samples from challenged and nonchallenged AMs were crosslinked with $1 \% \mathrm{wt} /$ vol formaldehyde for $10 \mathrm{~min}$ at room temperature and immediately quenched for 5 minutes with $125 \mathrm{mM}$ glycine at room temperature. The formaldehyde fixed samples were then sonicated to 150 to $500 \mathrm{bp}$ using a S220 (Covaris) and then ChIP-DNA was prepared using a manual chromatin immunoprecipitation method with an Antibody-Antigen incubation of 18 hours, followed by bead incubation for 135 minutes, and a 5-minute washing step repeated 6 times.

Approximately 0.5 million cells were used for each ChIP and approximately 50,000 cells for the input. We used H3K27ac antibody (Abcam, Ab4729, GR3211959-1). ChIP and Input libraries were prepared using the MicroPlex Library Preparation Kit (C05010012, Diagenode), with alterations including PCR enrichment (7-20 cycles) prior to size selection and use of Ampure beads for size selection (250-350 bp). Libraries for ChIP-Seq were sequenced on an Illumina NovaSeq 6000 S2 SR100.

Data availability. RNA-, ATAC-, and ChIP-Seq raw sequences and corresponding quantification matrices analyzed in this work were submitted to NCBI's Gene Expression Omnibus repository (GSE165709).

Statistics. Detailed description for raw sequences processing to statistical analyses are provided in the Supplemental Materials. Briefly, baseline and challenged transcriptome and epigenomic landscapes of AMs were determined following published protocols $(47,49)$. Gene expression readouts were generated using alignments and an annotated set of transcripts as input to Salmon (50). Differences in chromatin accessibility and H3K27ac levels were assessed with a count-based peak quantification as previously shown (51). Quantification files from each approach were merged as a single matrix, genes and peaks with low counts were filtered out, and libraries were normalized, scaled, and $\log _{2}$ transformed using edgeR and Limma-voom $(52,53)$.

For the statistical analysis, we defined linear models with blocking design on subject ID to account for interindividual differences and modelled the challenge effect nested within phenotypic groups. The use of a blocking design (i.e., using subject ID covariate as a main fixed effect) precluded the direct inclusion of covariates in the linear models. However, given the duplicated information for library pairs from a subject, a blocking design is effectively accounting for covariates in a nested fashion. For all experiments, we tested the challenge effect for HC, PrEP, and PLWH groups, while for RNA-Seq we also contrasted the phenotype groups against each other (interaction test). ATACSeq and ChIP-seq $P$ values were adjusted with Storey's false discovery rate (FDR) and peaks were considered significant if FDR was less than 0.05 and absolute log fold-change $\left(\log _{2} \mathrm{FC}\right)$ was greater than 0.2 . These were termed differentially open chromatin (DOC) for ATACSeq and differentially acetylated (DAc) when referring to ChIP-Seq. For RNA-Seq we employed a 2-stage FDR procedure and we considered genes to be DEGs if absolute $\log _{2} \mathrm{FC}$ was 0.2 or greater and stage $\mathrm{R}$ $P$ value was less than or equal to 0.05 (54).

We performed pathway and gene ontology enrichment analysis using 3 independent databases (KEGG, Reactome, and AmiGO). Enrichment results were merged and the Benjamini-Hochberg FDR method was applied to raw $P$ values. We tested TF motif enrichment in DOCs and DAc regions using HOMER (55). M. tuberculosis-triggered differences in TF footprint activity over DOC regions were estimated with HINT-ATAC (56). TF motifs with FDR less than 0.05 and activity score greater than 0.005 were considered significant. For plots displaying subject mean $\log _{2} \mathrm{FC}$ for genes in GO terms/pathways, we retrieved DEG IDs, which were part of significant pathway/GO terms, pooled the DEGs for each group, derived subject-wise $\log _{2} \mathrm{FC}$ for each gene, and averaged the $\log _{2} \mathrm{FC}$ from pooled genes per subject.

Study approval. The study was approved by the Research Ethics Board of the McGill University Health Center (MUHC) (MP-CUSM-15-406). All participants signed a written informed consent and were recruited at the McGill University Health Centre (MUHC, Montreal, Canada).

\section{Author contributions}

WCM analyzed the transcriptomic data and VMF analyzed the epigenomic data. ES, LBB, WCM, and VMF designed the study. $\mathrm{MO}$ and PC prepared samples and performed cell-based experi- 
ments for RNA-Seq, ATAC-Seq, and ChIP-Seq. AD, RHMS, and VY performed ChIP experiments. YZX carried out the cytokine quantification experiment. JPR and JG enrolled subjects. RO performed bronchoalveolar lavages. JS and AP provided analytical guidance and participated in initial data analyses. BK and CL provided experimental guidance for AM isolation from BAL samples. ES and LBB procured funding sources. WCM, VMF, LBB, and ES wrote the draft of the manuscript. All authors participated in the final assemble of the manuscript. ES conceptualized and supervised the project. WCM and VMF contributed equally to the manuscript. Co-first authorship order was established by type of analysis.

\section{Acknowledgments}

We thank all volunteers who participated in this study. We thank Pilar Domenech for help with M. tuberculosis cultures. We thank personnel of the CL3 platform of the RI-MUHC and the Centre d'expertise et de services Génome Québec. We thank all members of the Schurr and Barreiro labs for discussions of the data and helpful comments on the manuscript. This work was supported by grant 1R01AI124349 from the National Institutes of Health (to ES and LBB) and in part by the Fonds de la Recherche Québec-Santé (FRQ-S) Réseau SIDA/Maladies infectieuses and Thérapie cellulaire (to JPR). JS is supported through grants PID2019106859GA-IOO and RYC-2017-23560 from the Spanish Ministry of Science and Innovation (MICINN). This research was supported through a resource allocation in the Cedar high performance computing cluster by Compute Canada and WestGrid.

Address correspondence to: Erwin Schurr, Research Institute of the MUHC, 1001 Decarie Boulevard, Room EM3.3210, Montreal, H4A 3J1, Quebec, Canada. Email: erwin.schurr@mcgill.ca.
1. World Health Organization. Global Tuberculosis Report 2020. World Health Organization; 2020.

2. Lawn SD, Wilkinson RJ. ART and prevention of HIV-associated tuberculosis. Lancet HIV. 2015;2(6):e221-e222.

3. Narayanan S, et al. Impact of HIV infection on the recurrence of tuberculosis in South India. JInfect Dis. 2010;201(5):691-703.

4. Sonnenberg P, et al. HIV-1 and recurrence, relapse, and reinfection of tuberculosis after cure: a cohort study in South African mineworkers. Lancet. 2001;358(9294):1687-1693.

5. Crampin AC, et al. Recurrent TB: relapse or reinfection? The effect of HIV in a general population cohort in Malawi. AIDS. 2010;24(3):417-426.

6. Houben RM, et al. Human immunodeficiency virus associated tuberculosis more often due to recent infection than reactivation of latent infection. Int J Tuberc Lung Dis. 2011;15(1):24-31.

7. Cohen SB, et al. Alveolar macrophages provide an early mycobacterium tuberculosis niche and initiate dissemination. Cell Host Microbe. 2018;24(3):439-446.

8. Ackley SF, et al. Multiple exposures, reinfection and risk of progression to active tuberculosis. R Soc Open Sci. 2019;6(3):180999.

9. Simmons JD, et al. Immunological mechanisms of human resistance to persistent Mycobacterium tuberculosis infection. Nat Rev Immunol. 2018;18(9):575-589.

10. UNAIDS. UNAIDS Data 2019. www.unaids. org/sites/default/files/media_asset/2019UNAIDS-data_en.pdf. Updated 2019. Accessed June 28, 2021.

11. Barreiro LB, et al. Promoter variation in the DC-SIGN-encoding gene CD209 is associated with tuberculosis. PLoS Med. 2006;3(2):e20.

12. Martin MP, et al. Association of DC-SIGN promoter polymorphism with increased risk for parenteral, but not mucosal, acquisition of human immunodeficiency virus type 1 infection. J Virol. 2004;78(24):14053-14056.

13. Sakuntabhai A, et al. A variant in the CD209 promoter is associated with severity of dengue disease. Nat Genet. 2005;37(5):507-513.

14. Tailleux L, et al. DC-SIGN induction in alveolar macrophages defines privileged target host cells for mycobacteria in patients with tuberculosis. PLoS Med. 2005;2(12):e381.

15. Lugo-Villarino G, et al. The C-type lectin receptor DC-SIGN Has an anti-inflammatory role in human $\mathrm{M}(\mathrm{IL}-4)$ macrophages in response to Mycobacterium tuberculosis. Front Immunol. 2018;9:1123.

16. Zhang Z, et al. Acetylation-dependent deubiquitinase OTUD3 controls MAVS activation in innate antiviral immunity. Mol Cell. 2020;79(2):304-319.

17. Mack U, et al. LTBI: latent tuberculosis infection or lasting immune responses to M. tuberculosis? A TBNET consensus statement. Eur Respir J. 2009;33(5):956-973.

18. Wolf AJ, et al. Initiation of the adaptive immune response to Mycobacterium tuberculosis depends on antigen production in the local lymph node, not the lungs. J Exp Med. 2008;205(1):105-115.

19. Huang L, et al. Growth of Mycobacterium tuberculosis in vivo segregates with host macrophage metabolism and ontogeny. J Exp Med. 2018;215(4):1135-1152.

20. Rothchild AC, et al. Alveolar macrophages generate a noncanonical NRF2-driven transcriptional response to Mycobacterium tuberculosis in vivo. Sci Immunol. 2019;4(37): eaaw6693.

21. Kroon EE, et al. An observational study identifying highly tuberculosis-exposed, HIV-1-positive but persistently TB, tuberculin and IGRA negative persons with $\mathrm{M}$. tuberculosis specific antibodies in Cape Town, South Africa. EBioMedicine. 2020;61:103053.

22. Lu LL, et al. IFN-gamma-independent immune markers of Mycobacterium tuberculosis exposure. Nat Med. 2019;25(6):977-987.

23. Sonnenberg $\mathrm{P}$, et al. How soon after infection with HIV does the risk of tuberculosis start to increase? A retrospective cohort study in South African gold miners. JInfect Dis. 2005;191(2):150-158.

24. Gupta A, et al. Tuberculosis incidence rates during 8 years of follow-up of an antiretroviral treatment cohort in South Africa: comparison with rates in the community. PLoS One. 2012;7(3):e34156.

25. Jambo KC, et al. Small alveolar macrophages are infected preferentially by HIV and exhibit impaired phagocytic function. Mucosal Immunol. 2014;7(5):1116-1126.

26. Gordon MA, et al. Primary macrophages from HIV-infected adults show dysregulated cytokine responses to Salmonella, but normal internalization and killing. AIDS. 2007;21(18):2399-2408.

27. Yeligar SM, et al. Dysregulation of alveolar macrophage PPAR $\gamma, \mathrm{NADPH}$ oxidases, and TGF $\beta_{1}$ in otherwise healthy HIV-infected individuals. AIDS Res Hum Retroviruses. 2017;33(10):1018-1026.

28. Collini PJ, et al. HIV gp120 in the lungs of antiretroviral therapy-treated individuals impairs alveolar macrophage responses to pneumococci. $\mathrm{Am} \mathrm{J}$ Respir Crit Care Med. 2018;197(12):1604-1615.

29. Cribbs SK, et al. Healthy HIV-1-infected individuals on highly active antiretroviral therapy harbor HIV-1 in their alveolar macrophages. AIDS Res Hum Retroviruses. 2015;31(1):64-70.

30. Costiniuk CT, et al. HIV persistence in mucosal CD4+ T cells within the lungs of adults receiving long-term suppressive antiretroviral therapy. AIDS. 2018;32(16):2279-2289.

31. Crothers K, et al. HIV infection and risk for incident pulmonary diseases in the combination antiretroviral therapy era. Am J Respir Crit Care Med. 2011;183(3):388-395.

32. Gingo MR, et al. Pulmonary symptoms and diagnoses are associated with HIV in the MACS and WIHS cohorts. BMC Pulm Med. 2014;14:75.

33. Cribbs SK, et al. Characteristics and outcomes of HIV-infected patients with severe sepsis: continued risk in the post-highly active antiretroviral therapy era. Crit Care Med. 2015;43(8):1638-1645.

34. Bhaskaran K, et al. HIV infection and COVID-19 death: a population-based cohort analysis of UK primary care data and linked national death registrations within the OpenSAFELY platform. Lancet HIV . 2021;8(1):e24-e32.

35. Tesoriero JM, et al. COVID-19 outcomes among persons living with or without diagnosed HIV infection in New York State. JAMA Netw Open. 2021;4(2):e2037069.

36. Grant RA, et al. Circuits between infected macrophages and T cells in SARS-CoV-2 pneumonia. Nature. 2021;590(7847):635-641.

37. Muller JT, Al Khalili Y. Emtricitabine. In: Stat- 
Pearls. StatPearls Publishing; 2021:NBK539853.

38. Tetteh RA, et al. Pre-exposure prophylaxis for HIV prevention: safety concerns. Drug Saf. 2017;40(4):273-283.

39. Mayer KH, et al. Emtricitabine and tenofovir alafenamide vs emtricitabine and tenofovir disoproxil fumarate for HIV pre-exposure prophylaxis (DISCOVER): primary results from a randomised, double-blind, multicentre, active-controlled, phase 3, non-inferiority trial. Lancet. 2020;396(10246):239-254.

40. Cohen J, et al. Astrocyte senescence and metabolic changes in response to HIV antiretroviral therapy drugs. Front Aging Neurosci. 2017;9:281.

41. Cohen J, et al. HIV antiretroviral therapy drugs induce premature senescence and altered physiology in HUVECs. Mech Ageing Dev. 2018;175:74-82.

42. Khan N, et al. M. tuberculosis reprograms hematopoietic stem cells to limit myelopoiesis and impair trained immunity. Cell. 2020;183(3):752-770.

43. Yao Y, et al. Induction of autonomous memory alveolar macrophages requires $\mathrm{T}$ cell help and is critical to trained immunity. Cell. 2018;175(6):1634-1650.

44. Meziane O, et al. Peculiar phenotypic and cytotoxic features of pulmonary mucosal CD8 $\mathrm{T}$ cells in people living with HIV receiving long-term antiretroviral therapy. Jimmunol. 2021;206(3):641-651.

45. Jansen K, et al. STI in times of PrEP: high prevalence of chlamydia, gonorrhea, and mycoplasma at different anatomic sites in men who have sex with men in Germany. BMC Infect Dis. 2020;20(1):110.

46. Meyer KC, et al. An official American Thoracic Society clinical practice guideline: the clinical utility of bronchoalveolar lavage cellular analysis in interstitial lung disease. Am J Respir Crit Care Med. 2012;185(9):1004-1014.

47. Buenrostro JD, et al. Transposition of native chromatin for fast and sensitive epigenomic profiling of open chromatin, DNA-binding proteins and nucleosome position. Nat Methods. 2013;10(12):1213-1218.

48. Buenrostro JD, et al. ATAC-seq: a method for assaying chromatin accessibility genome-wide. Curr Protoc Mol Biol. 2015;109:21.9.1-21.29.9.

49. Pacis A, et al. Bacterial infection remodels the DNA methylation landscape of human dendritic cells. Genome Res. 2015;25(12):1801-1811.
50. Patro R, et al. Salmon provides fast and biasaware quantification of transcript expression. Nat Methods. 2017;14(4):417-419.

51. Corces MR, et al. The chromatin accessibility landscape of primary human cancers. Science. 2018;362(6413):eaav1898.

52. Robinson MD, et al. edgeR: a Bioconductor package for differential expression analysis of digital gene expression data. Bioinformatics. 2010;26(1):139-140.

53. Law CW, et al. voom: Precision weights unlock linear model analysis tools for RNA-seq read counts. Genome Biol. 2014;15(2):R29.

54. Van den Berge K, et al. stageR: a general stagewise method for controlling the gene-level false discovery rate in differential expression and differential transcript usage. Genome Biol. 2017;18(1):151.

55. Heinz S, et al. Simple combinations of lineagedetermining transcription factors prime cisregulatory elements required for macrophage and B cell identities. Mol Cell. 2010;38(4):576-589.

56. Li Z, et al. Identification of transcription factor binding sites using ATAC-seq. Genome Biol. 2019;20(1):45. 